\title{
Combinatorial group theory and the homotopy groups of finite complexes
}

\author{
ROMAN MIKHAILOV \\ JIE WU
}

\begin{abstract}
For $n>k \geq 3$, we construct a finitely generated group with explicit generators and relations obtained from braid groups, whose center is exactly $\pi_{n}\left(S^{k}\right)$. Our methods can be extended to obtain combinatorial descriptions of homotopy groups of finite complexes. As an example, we also give a combinatorial description of the homotopy groups of Moore spaces.
\end{abstract}

55Q40, 55Q52; 18G30, 20E06, 20F36, 55U10, 57M07

\section{Introduction}

Homotopy groups of spheres play a central role in the algebraic topology. The traditional approaches to understanding homotopy groups of spheres include Adams spectral sequence, EHP-sequences, Toda secondary operations, $J$-homomorphisms, connections between homotopy theory and cobordisms etc.

A description of homotopy groups of the 2-dimensional sphere in terms of combinatorial group theory was discovered by the second author in 1994 and given in his thesis [22], with a published version in [24]. In this article we give a combinatorial description of the homotopy groups of $k$-dimensional spheres with $k \geq 3$. The description is given by identifying the homotopy groups as the center of a quotient group of the self free products with amalgamation of pure braid groups by certain canonical subgroups (Theorem 2.2; see Section 2 for the explicit construction). Our methods can be extended to obtain combinatorial descriptions of homotopy groups of finite complexes. As an example, we also give a combinatorial description of the homotopy groups of Moore spaces.

We follow earlier approaches in the use of simplicial groups to study these questions. The notions of simplicial sets and simplicial groups have been widely studied since they were introduced in the early of 1950s when D Kan established the foundational work for simplicial homotopy theory $[13 ; 14]$. Various important results have been achieved by studying simplicial groups. For instance, a special case of the Adams 
spectral sequence can be obtained from the lower central series of Kan's construction [4] for computing homotopy groups. The results of the paper [24] produced surprising connections between group theory, group ring theory, homotopy and low-dimensional topology. For example, the authors and Passi applied homotopy groups of spheres to describe the subgroups determined by ideals in group rings in [18]. The results from [24] play a central role in connecting the homotopy groups of $S^{2}$ with Brunnian braids on the disk and sphere in the paper [3] by Berrick, Cohen, Wong and Wu. The main result in [24] was generalized by Ellis and Mikhailov in [11] by studying a van Kampen-type theorem for higher homotopy groups. Serious study of Brunnian braids by the authors along with Bardakov and Vershinin [1], and $\mathrm{Li}$ and $\mathrm{Wu}$ [16] introduced the notion of symmetric commutator subgroups in determining the group of Brunnian braids on surfaces $S$ for $S \neq S^{2}$ or $\mathbb{R} \mathrm{P}^{2}$. By using this notion together with the embedding theorem due to Cohen and Wu in [7, Theorem 1.2] as well as the Whitehead Theorem on free products with amalgamation of simplicial groups (Kan and Thurston [15, Proposition 4.3]), we are able to control the Moore boundaries of our simplicial group models for the loop spaces of spheres and Moore spaces using a different cone derived from the pure braid complex, which leads to our results.

In this paper we exhibit new connections between homotopy theory and group theory. The braids enter this context in the following three important ways:

(1) The contractible property of the simplicial group $\mathrm{AP}_{*}$ introduced by Cohen and Wu in $[7 ; 8]$ with $n$-simplices given by the $(n+1)$-strand pure braid group plays an essential role for determining the homotopy type of simplicial groups given by the free product with amalgamations of copies of $\mathrm{AP}_{*}$.

(2) The Moore chains and the Moore boundaries of free products with amalgamations of copies of $\mathrm{AP}_{*}$ can be determined, where the simplicial group $\mathcal{G}$ was technically introduced in Section 4.4 as a free simplicial group deformation of $\mathrm{AP}_{*}$ (forgetting defining relations) for the determination of the Moore chains and Moore boundaries.

(3) The homotopy groups of higher dimensional spheres can be described in terms of free product with amalgamations of $\mathrm{AP}_{*}$.

The article is organized as follows. In Section 2, we recall the description of the homotopy groups of the 2-dimensional sphere from [24] and formulate the main results of this paper. In particular, the complete description of groups such that their centers are homotopy groups is given. In Section 3, we study free products with amalgamation of simplicial groups. In some cases, these products present simplicial models for loop spaces of homotopy push-out spaces. In Section 4 , for $k \geq 3$, we construct simplicial 
groups such that their geometrical realization is homotopy equivalent to the loops on the $k$-dimensional sphere. There is a natural way to describe Moore boundaries of these simplicial groups and this description is a key point in the proof of Theorem 2.2, which we give in Section 4. In Section 5, we consider triple free products with amalgamation of simplicial braid groups and construct simplicial models for loop spaces for Moore spaces. For $k \geq 3$, we give a description of a finitely generated group such that its center is the $n^{\text {th }}$ homotopy group of the $k$-dimensional $\mathbb{Z} / q$-Moore space (Theorem 5.4). Section 6 is about 3-dimensional Moore spaces. In this case, the simplicial models for loop spaces of Moore spaces can be simplified. We prove Theorem 2.3 in Section 6 .

\section{Preliminaries and formulations of main results}

Recall briefly the combinatorial description of $\pi_{*}\left(S^{2}\right)$ from $[22 ; 24]$. Let

$$
F_{n}=\left\langle x_{0}, x_{1}, \ldots, x_{n} \mid x_{0} x_{1} \cdots x_{n}\right\rangle
$$

be the one-relator group generated by $x_{0}, \ldots, x_{n}$ with the defining relation $x_{0} \cdots x_{n}=$ 1. (Note that $F_{n}$ is a free group of rank $n$ with a basis given by $\left\{x_{1}, \ldots, x_{n}\right\}$.) Let $R_{i}=\left\langle x_{i}\right\rangle^{F_{n}}$ be the normal closure of $x_{i}$ in $F_{n}$ for $0 \leq i \leq n$. We can form a symmetric commutator subgroup

$$
\left[R_{0}, R_{1}, \ldots, R_{n}\right]_{S}=\prod_{\sigma \in \Sigma_{n+1}}\left[\ldots\left[R_{\sigma(0)}, R_{\sigma(1)}\right], \ldots, R_{\sigma(n)}\right]
$$

where the symmetric group $\Sigma_{n+1}$ acts on $\{0,1, \ldots, n\}$. The symmetric group $\Sigma_{n+1}$ permutes the indices of the subgroups $R_{i}$. There is an action of the braid group $B_{n+1}$ on $F_{n}=\left\langle x_{0}, x_{1}, \ldots, x_{n} \mid x_{0} x_{1} \cdots x_{n}\right\rangle$ by the Artin representation, which induces an action of $B_{n+1}$ on the quotient group $F_{n} /\left[R_{0}, R_{1}, \ldots, R_{n}\right]_{S}$. By Wu [25, Theorem 1.2], the center of $F_{n} /\left[R_{0}, R_{1}, \ldots, R_{n}\right]_{S}$ is exactly given by the fixed set of the pure braid group $P_{n+1}$ action on $F_{n} /\left[R_{0}, R_{1}, \ldots, R_{n}\right]_{S}$ for $n \geq 3$. This gives an explicit subgroup of $F_{n}$ with a set of generators that can be understood by taking a collection of iterated commutators. By [24, Theorem 1.4], we have the following combinatorial description of $\pi_{*}\left(S^{2}\right)$.

Theorem 2.1 For $n \geq 1$, there is an isomorphism

$$
\pi_{n+1}\left(S^{2}\right) \cong \frac{R_{0} \cap \cdots \cap R_{n}}{\left[R_{0}, \ldots, R_{n}\right]_{S}}
$$

This quotient group is isomorphic to the center of the group $F_{n} /\left[R_{0}, R_{1}, \ldots, R_{n}\right]_{S}$. 
The groups $F_{n} /\left[R_{0}, R_{1}, \ldots, R_{n}\right]_{S}$ can be defined using explicit generators and relations. This situation is very interesting from the group-theoretical point of view: we do not know how to describe the homotopy groups $\pi_{*}\left(S^{2}\right)$ in terms of generators and relations, but we can describe a bigger group whose center is exactly $\pi_{*}\left(S^{2}\right)$. Philosophically speaking, the center is the most elementary natural, characteristic Abelian subgroup of a group. There is a lot of work on centers of groups. For example, people have studied the question of how to realize a given Abelian group as the center of a finitely generated or finitely presented group; see Baumslag [2] and Houcine [12]. For a group $H$, we denote it center by $Z(H)$. Centers of groups appear in different places of low-dimensional homotopy theory. For example, let $G$ be a group with trivial center. Then there is a natural isomorphism

$$
\pi_{3}(\Sigma K(G, 1)) \cong Z(G \otimes G)
$$

where $G \otimes G$ is the non-Abelian tensor square in the sense of Brown and Loday [5]. Let $K$ be a 2-dimensional cell complex, $K^{1}$ its $1-$ skeleton; then there is a natural isomorphism

$$
\pi_{2}(K) \cong Z\left(\pi_{2}\left(K, K^{1}\right)\right) .
$$

It has been the concern of many people whether one can give a combinatorial description of the homotopy groups of higher dimensional spheres, ever since Theorem 2.1 was announced in 1994. Technically the proof of this theorem was obtained by determining the Moore boundaries of Milnor's $F[K]$-construction [19] on the simplicial 1-sphere $S^{1}$, which is a simplicial group model for $\Omega S^{2}$. A canonical approach is to study Milnor's construction $F\left[S^{k}\right] \simeq \Omega S^{k+1}$ for $k>1$. Although there have been some attempts by Zhao and Wang [26] to study this question using $F\left[S^{k}\right]$, technical difficulties arise in handling Moore boundaries of $F\left[S^{k}\right]$ in a good way, and combinatorial descriptions of the homotopy groups of higher dimensional spheres using the simplicial group model $F\left[S^{k}\right]$ would be very messy.

In this article, we give a combinatorial description of $\pi_{*}\left(S^{k}\right)$ for any $k \geq 3$ by using the free product with amalgamation of pure braid groups. Our construction is as follows. Given $k \geq 3, n \geq 2$, let $P_{n}$ be the $n$-strand Artin pure braid group with the standard generators $A_{i, j}$ for $1 \leq i<j \leq n$. We construct a (free) subgroup $Q_{n, k}$ of $P_{n}$ from cabling as follows. Our cabling process starts from $P_{2}=\mathbb{Z}$ generated by the 2 -strand pure braid $A_{1,2}$.

Step 1 Consider the 2-strand pure braid $A_{1,2}$. Let $\xi_{i}$ be $(k-1)$-strand braid obtained by inserting $i$ parallel strands into the tubular neighborhood of the first strand 
of $A_{1,2}$ and $k-i-1$ parallel strands into the tubular neighborhood of the second strand of $A_{1,2}$ for $1 \leq i \leq k-2 .^{1}$

Step 2 Let $\alpha_{k}=\left[\ldots\left[\left[\xi_{1}^{-1}, \xi_{1} \xi_{2}^{-1}\right], \xi_{2} \xi_{3}^{-1}\right], \ldots, \xi_{k-3} \xi_{k-2}^{-1}, \xi_{k-2}\right]$ be a fixed choice of $(k-1)-$ strand braid, which is a nontrivial $(k-1)-$ strand Brunnian braid. ${ }^{2}$

Step 3 By applying the cabling process as in Step 1 to the element $\alpha_{k}$, we insert parallel strands into the tubular neighborhood of the strands of $\alpha_{k}$ in any possible way to obtain $n$-strand braids. As the order in which the strands are inserted is arbitrary, there are $\left(\begin{array}{l}n-1 \\ k-2\end{array}\right)$ ways of doing this. Label the $\left(\begin{array}{l}n-1 \\ k-2\end{array}\right)$ $n$-strand braids obtained in this way by $y_{j}$ for $1 \leq j \leq\left(\begin{array}{l}n-1 \\ k-2\end{array}\right)$.

Let $Q_{n, k}$ be the subgroup of $P_{n}$ generated by $y_{j}$ for $1 \leq j \leq\left(\begin{array}{l}n-1 \\ k-2\end{array}\right)$. Now consider the free product with amalgamation

$$
P_{n} * Q_{n, k} P_{n}
$$

Namely this amalgamation is obtained by identifying the elements $y_{j}$ in two copies of $P_{n}$. Let $A_{i, j}$ be the generators for the first copy of $P_{n}$ and let $A_{i, j}^{\prime}$ denote the generators $A_{i, j}$ for the second copy of $P_{n}$. Let

$$
R_{i, j}=\left\langle A_{i, j}, A_{i, j}^{\prime}\right\rangle^{P_{n} * Q_{n, k} P_{n}}
$$

be the normal closure of $A_{i, j}, A_{i, j}^{\prime}$ in $P_{n} * Q_{n, k} P_{n}$. Let

$$
\left[R_{i, j} \mid 1 \leq i<j \leq n\right]_{S}=\prod_{\{1,2, \ldots, n\}=\left\{i_{1}, j_{1}, \ldots, i_{t}, j_{t}\right\}}\left[\ldots\left[R_{i_{1}, j_{1}}, R_{i_{2}, j_{2}}\right], \ldots, R_{i_{t}, j_{t}}\right]
$$

be the product of all commutator subgroups such that each integer $1 \leq j \leq n$ appears as one of indices at least once. Our main theorem is as follows:

Theorem 2.2 Let $k \geq 3$. The homotopy group $\pi_{n}\left(S^{k}\right)$ is isomorphic to the center of the group

$$
\left(P_{n} * Q_{n, k} P_{n}\right) /\left[R_{i, j} \mid 1 \leq i<j \leq n\right]_{S}
$$

for any $n$ if $k>3$ and any $n \neq 3$ if $k=3$.

Note The only exceptional case is $k=3$ and $n=3$. In this case, $\pi_{3}\left(S^{3}\right)=\mathbb{Z}$ while the center of the group is bigger than $\mathbb{Z}$.

${ }^{1}$ The braids $\xi_{i}$ were introduced in [7;8] with notation of $x_{i}$. A formula for $\xi_{i}$ in terms of the $A_{i, j}$ Artin's generators was given in [7, Formula 1 in the proof of Lemma 2.2].

${ }^{2}$ For a group $G$ and $g, h \in G$, we use the notation $[g, h]:=g^{-1} h^{-1} g h$. 
The center of the group $\left(P_{n} * Q_{n, k} P_{n}\right) /\left[R_{i, j} \mid 1 \leq i<j \leq n\right]_{S}$ is in fact given by Brunnian words in the following sense: Let $\bar{d}_{k}: P_{n} \rightarrow P_{n-1}$ be the operation of removing the $k^{\text {th }}$ strand for $1 \leq k \leq n$. A Brunnian braid means an $n$-braid $\beta$ such that $\bar{d}_{k} \beta=1$ for any $1 \leq k \leq n$. Namely, $\beta$ becomes a trivial braid after removing any one of its strands. This notion can be canonically extended to free products of braid groups. In other words, we have a canonical operation $\bar{d}_{k}: P_{n} * P_{n} \rightarrow P_{n-1} * P_{n-1}$ given by removing the $k^{\text {th }}$ strand. A Brunnian word in $P_{n} * P_{n}$ means a word $w$ such that $\bar{d}_{k} w=1$ for any $1 \leq k \leq n$. It can be seen from our techniques that the Brunnian words in $P_{n} * P_{n}$ are exactly given by the symmetric commutator subgroup $\left[R_{i, j} \mid 1 \leq i<j \leq n\right]_{S}$. However the question on determining Brunnian words in free products of braid groups with amalgamations becomes very tricky. The question here is about the self free product of $P_{n}$ with the amalgamation given by the subgroup $Q_{n, k}$. It is straightforward to check that the strand-removing operation $\bar{d}_{k}$ maps $Q_{n, k}$ into $Q_{n-1, k}$ and so the removing operation $\bar{d}_{k}: P_{n} * Q_{n, k} P_{n} \rightarrow P_{n-1} * Q_{n-1, k} P_{n-1}$ is a well-defined group homomorphism. From our construction of simplicial groups given by free products with amalgamation, the Brunnian words in $P_{n} * Q_{n, k} P_{n}$ are exactly the Moore cycles in our simplicial group model for $\Omega S^{k}$ and, from Theorem 2.2, the center

$$
Z\left(\left(P_{n} * Q_{n, k} P_{n}\right) /\left[R_{i, j} \mid 1 \leq i<j \leq n\right]_{S}\right) \cong \pi_{n}\left(S^{k}\right)
$$

is exactly given by the Brunnian words in $P_{n} * Q_{n, k} P_{n}$ modulo the subgroup $\left[R_{i, j} \mid 1 \leq i<j \leq n\right]_{S}$. One important point concerning Brunnian words of the self free product with amalgamation of $P_{n}$ is that the homotopy groups $\pi_{n}\left(S^{k}\right)$ can be given as quotient groups of Brunnian subgroups for any $k \geq 3$.

Mark Mahowald asked in 1995 whether one can give a combinatorial description of the homotopy groups of the suspensions of real projective spaces. In this article, we also give a combinatorial description of the homotopy groups of Moore spaces as the first step for attacking Mahowald's question. Let $M(\mathbb{Z} / q, k)$ be the $(k+1)$-dimensional Moore space. Namely $M(\mathbb{Z} / q, k)=S^{k} \cup_{q} e^{k+1}$ is the homotopy cofibre of the degree $q$ map $S^{k} \rightarrow S^{k}$. If $k \geq 3$, we give a combinatorial description of $\pi_{*}(M(\mathbb{Z} / q, k))$ given as the centers of quotient groups of threefold self free product with amalgamation of pure braid groups, which is similar to the description given in Theorem 2.2. (The detailed description will be given in Section 5.) This description is less explicit then the one given in Theorem 2.2, but it leads to combinatorial descriptions of homotopy groups of finite complexes from iterated self free products with amalgamations of pure braid groups.

For the homotopy groups of 3-dimensional Moore spaces, there is an explicit combinatorial description that deserves to be described here as it arises in certain divisibility 
questions concerning braids. Let $\xi_{1}, \ldots, \xi_{n-1}$ be $n$-strand braid obtained by cabling $A_{1,2}$ as described in Step 1 of the construction for the group $Q_{n, k}$. It was proved in [7] that the subgroup of $P_{n}$ generated by $\xi_{1}, \ldots, \xi_{n-1}$ is a free group of rank $n-1$ with a basis given by $\xi_{1}, \ldots, \xi_{n-1}$. Let $F_{n-1}=\left\langle\xi_{1}, \ldots, \xi_{n-1}\right\rangle \leq P_{n}$ be the subgroup generated by $\xi_{1}, \ldots, \xi_{n-1}$. Given an integer $q$, since $F_{n-1}=\left\langle\xi_{1}, \ldots, \xi_{n-1}\right\rangle$ is free, there is a group homomorphism $\phi_{q}: F_{n-1} \rightarrow F_{n-1}$ such that $\phi_{q}\left(\xi_{j}\right)=\xi_{j}^{q}$ for $1 \leq j \leq n-1$. Now we form a free product with amalgamation by the push-out diagram

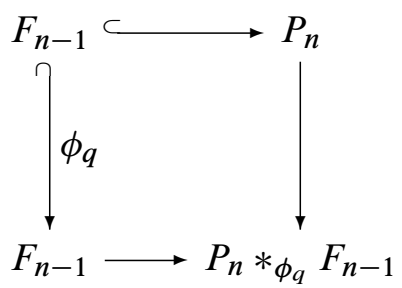

namely the group $P_{n} *_{\phi_{q}} F_{n-1}$, which is the free product given by identifying the subgroup $F_{n-1}$ with the subgroup of $F_{n-1}$ generated by $\xi_{1}^{q}, \ldots, \xi_{n-1}^{q}$ in a canonical way. Let $y_{j}$ denote the generator $\xi_{j}$ for $F_{n-1}$ as the second factor in the free product $P_{n} * \phi_{q} F_{n-1}$ for $1 \leq j \leq n-1$. Let

$$
R_{1}=\left\langle y_{1}\right\rangle^{P_{n} *_{\phi_{q}} F_{n-1}}, \quad R_{j}=\left\langle y_{j-1} y_{j}^{-1}\right\rangle^{P_{n} *_{\phi q} F_{n-1}}, \quad R_{n}=\left\langle y_{n-1}\right\rangle^{P_{n} *_{\phi_{q}} F_{n-1}}
$$

be the normal closure of $y_{1}, y_{j-1} y_{j}^{-1}, y_{n-1}$ in $P_{n} *_{\phi_{q}} F_{n-1}$, respectively, for $2 \leq j \leq$ $n-1$. Let

$$
R_{S, t}=\left\langle A_{s, t}\right\rangle^{P_{n} *_{\phi_{q}} F_{n-1}}
$$

be the normal closure of $A_{s, t}$ in $P_{n} *_{\phi_{q}} F_{n-1}$ for $1 \leq s<t \leq n$. Define the index set $\operatorname{Index}\left(R_{j}\right)=\{j\}$ for $1 \leq j \leq n$ and $\operatorname{Index}\left(R_{s, t}\right)=\{s, t\}$ for $1 \leq s<t \leq n$. Now define the symmetric commutator subgroup

$$
\left[R_{i}, R_{s, t} \mid 1 \leq i \leq n, 1 \leq s<t \leq n\right]_{S}=\underset{\{1,2, \ldots, n\}=\bigcup_{j=1}^{t} \operatorname{Index}\left(C_{j}\right)}{\left[\ldots\left[C_{1}, C_{2}\right], \ldots, C_{t}\right],}
$$

where each $C_{j}=R_{i}$ or $R_{s, t}$ for some $i$ or $(s, t)$.

Theorem 2.3 The homotopy group $\pi_{n}(M(\mathbb{Z} / q, 2))$ is isomorphic to the center of the group

$$
\left(P_{n} * \phi_{q} F_{n-1}\right) /\left[R_{i}, R_{s, t} \mid 1 \leq i \leq n, 1 \leq s<t \leq n\right]_{S}
$$

for $n \neq 3$. 
Note For the exceptional case $n=3, \pi_{3}(M(\mathbb{Z} / q, 2))$ is contained in the center but the equality fails.

\section{Free products with amalgamation on simplicial groups}

Let $\phi: G \rightarrow G^{\prime}$ and $\psi: G \rightarrow G^{\prime \prime}$ be group monomorphisms. Then we have the free product with amalgamation $G^{\prime}{ }^{*} G G^{\prime \prime}$. More precisely $G^{\prime} *_{G} G^{\prime \prime}$ is the quotient group of the free product $G^{\prime} * G^{\prime \prime}$ by the normal closure of the elements $\phi(g) \psi(g)^{-1}$ for $g \in G$. The group $G^{\prime} *_{G} G^{\prime \prime}$ has the universal property that the following diagram

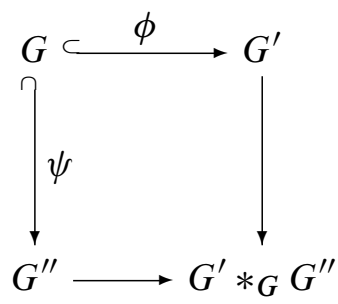

is a pushout diagram in the category of groups. Let $G^{\prime}=\left\langle X^{\prime} \mid R^{\prime}\right\rangle$ and $G^{\prime \prime}=\left\langle X^{\prime \prime} \mid R^{\prime \prime}\right\rangle$ be presentations of the groups $G^{\prime}$ and $G^{\prime \prime}$, respectively. Let $X$ be a set of generators for the group $G$. Then the group $G^{\prime} *_{G} G^{\prime \prime}$ has a presentation

$$
\left.G^{\prime} *_{G} G^{\prime \prime}=\left\langle X^{\prime}, X^{\prime \prime}\right| R^{\prime}, R^{\prime \prime}, \phi(x) \psi(x)^{-1} \text { for } x \in X\right\rangle .
$$

In particular, if $X^{\prime}, X^{\prime \prime}, R^{\prime}, R^{\prime \prime}$ and $X$ are finite sets, then $G^{\prime} *_{G} G^{\prime \prime}$ is a finitely presented group with a presentation given as above. The notion of free product with amalgamation can be canonically extended to the category of simplicial groups.

Recall that a simplicial group $G$ consists in a sequence of groups $G=\left\{G_{n}\right\}_{n \geq 0}$ with face homomorphisms $d_{i}: G_{n} \rightarrow G_{n-1}$ and degeneracy homomorphisms $s_{i}: G_{n} \rightarrow$ $G_{n+1}$ for $0 \leq i \leq n$ such that the following simplicial identities hold:

(1) $\Delta$-identity: $d_{i} d_{j}=d_{j} d_{i+1}$ for $i \geq j$

(2) Degeneracy Identity: $s_{i} s_{j}=s_{j+1} s_{i}$ for $i \leq j$

(3) Mixing Relation:

$$
d_{i} s_{j}= \begin{cases}s_{j-1} d_{i} & \text { if } i<j \\ \text { id } & \text { if } i=j, j+1 \\ s_{j} d_{i-1} & \text { if } i>j+1\end{cases}
$$

A simplicial homomorphism $f: G \rightarrow G^{\prime}$ consists in a sequence of group homomorphisms $f=\left\{f_{n}\right\}$ with $f_{n}$ : $G_{n} \rightarrow G_{n}^{\prime}$ such that $d_{i}^{G^{\prime}} f_{n}=f_{n-1} d_{i}^{G}$ and $s_{i}^{G^{\prime}} f_{n}=f_{n+1} s_{i}^{G}$ 
for $0 \leq i \leq n$. A simplicial monomorphism $f: G \rightarrow G^{\prime}$ means a simplicial homomorphism $f=\left\{f_{n}\right\}$ such that each $f_{n}: G_{n} \rightarrow G_{n}^{\prime}$ is a monomorphism. Similarly we have the notion of simplicial epimorphism.

For a simplicial group $G$, recall that the Moore chain complex $N_{*} G$ is defined by

$$
N_{n} G=\bigcap_{i=1}^{n} \operatorname{Ker}\left(d_{i}: G_{n} \rightarrow G_{n-1}\right)
$$

with the differential given by the restriction of the first face $d_{0} \mid: N_{n} G \rightarrow N_{n-1} G$. The Moore chain complex functor has the following important properties. For a simplicial set $X$, let $|X|$ denote its geometric realization.

Proposition 3.1 The following statements hold:

(1) Let $G$ be any simplicial group. Then there is a natural isomorphism

$$
H_{n}\left(N_{*} G ; d_{0} \mid\right) \cong \pi_{n}(|G|)
$$

for all $n$.

(2) Let $f: G \rightarrow G^{\prime}$ be a simplicial homomorphism. Then $f$ is a simplicial monomorphism (epimorphism) if and only if

$$
N(f): N_{q} G \longrightarrow N_{q} G^{\prime}
$$

is a monomorphism (epimorphism) for all $q$.

(3) A sequence of simplicial groups

$$
1 \rightarrow G^{\prime} \rightarrow G \rightarrow G^{\prime \prime} \rightarrow 1
$$

is short exact if and only if the corresponding sequence of Moore chain complexes

$$
1 \rightarrow N_{*} G^{\prime} \rightarrow N_{*} G \rightarrow N_{*} G^{\prime \prime} \rightarrow 1
$$

is short exact.

Proof Assertion (1) is the classical theorem of John Moore; see the survey paper by Curtis [10]. Assertion (2) is given in Quillen's book [20, Lemma 5, 3.8].

(3) By [3, Proposition 4.1.4], the Moore chain functor is an exact functor. We show that the inverse statement is also true. Namely if $1 \rightarrow N_{*} G^{\prime} \rightarrow N_{*} G \rightarrow N_{*} G^{\prime \prime} \rightarrow 1$ is short exact, then $1 \rightarrow G^{\prime} \rightarrow G \rightarrow G^{\prime \prime} \rightarrow 1$ is short exact. By assertion (2), $G^{\prime} \rightarrow G$ is a simplicial monomorphism and $G \rightarrow G^{\prime \prime}$ is a simplicial epimorphism. From Conduché's 
decomposition theorem of simplicial groups [9], the composite $G^{\prime} \rightarrow G \rightarrow G^{\prime \prime}$ is trivial and so $G^{\prime}$ is mapped into $\operatorname{Ker}\left(G \rightarrow G^{\prime \prime}\right)$. Since

$$
N_{*}\left(G^{\prime}\right) \cong N_{*} \operatorname{Ker}\left(G \rightarrow G^{\prime \prime}\right)=\operatorname{Ker}\left(N_{*} G \rightarrow N_{*} G^{\prime \prime}\right),
$$

$G^{\prime} \rightarrow \operatorname{Ker}\left(G \rightarrow G^{\prime \prime}\right)$ is an isomorphism by assertion (2) and the result follows.

Let $\mathcal{Z}_{n} G=\bigcap_{i=0}^{n} \operatorname{Ker}\left(d_{i}: G_{n} \rightarrow G_{n-1}\right) \leq N_{n} G$ be the Moore cycles and let $\mathcal{B}_{n} \mathcal{G}=$ $d_{0}\left(N_{n+1} G\right) \leq \mathcal{Z}_{n} G$ be the Moore boundaries. By assertion (1), the homotopy group $\pi_{n}(|G|)$ is given by $\mathcal{Z}_{n} G / \mathcal{B}_{n} G$.

The construction of free product with amalgamation on simplicial groups is given in the same way. Let $\phi: G \rightarrow G^{\prime}$ and $\psi: G \rightarrow G^{\prime \prime}$ be simplicial monomorphisms. Then $G^{\prime} *_{G} G^{\prime \prime}$ is a simplicial group where each $\left(G^{\prime} *_{G} G^{\prime \prime}\right)_{n}$ is the free product with amalgamation of $G_{n}^{\prime} * G_{n} G_{n}^{\prime \prime}$ for the group homomorphisms $\phi_{n}: G_{n} \rightarrow G_{n}^{\prime}$ and $\psi_{n}: G_{n} \rightarrow G_{n}^{\prime \prime}$. The face homomorphisms are (uniquely) determined by the pushout property:

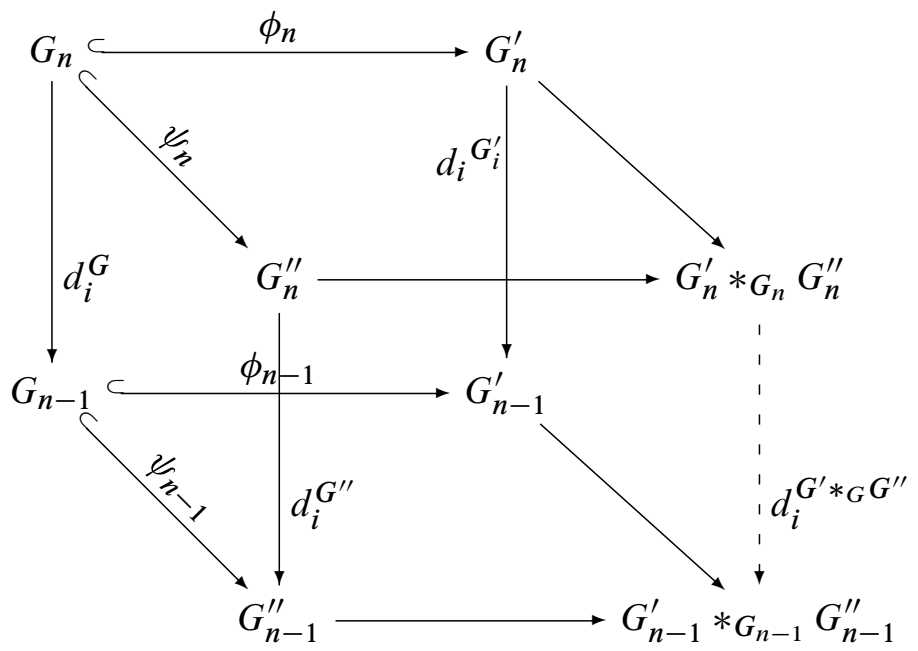

Similarly the degeneracy homomorphisms are (uniquely) determined by the pushout property. The uniqueness of the induced face and degeneracy homomorphisms forces the simplicial identities to hold for $d_{i}^{G^{\prime} *_{G} G^{\prime \prime}}$ and $s_{j}^{G^{\prime} *_{G} G^{\prime \prime}}$ and so $G^{\prime} *_{G} G^{\prime \prime}$ becomes a simplicial group. If we write the elements $w$ in

$$
\left(G^{\prime} *_{G} G^{\prime \prime}\right)_{n}=G_{n}^{\prime} *_{G_{n}} G_{n}^{\prime \prime}
$$

in terms of words as a product of elements from $G_{n}^{\prime}$ or $G_{n}^{\prime \prime}$, then $d_{i}^{G^{\prime} *_{G} G^{\prime \prime}}(w)$ is given by applying $d_{i}^{G^{\prime}}$ or $d_{i}^{G^{\prime \prime}}$ to the factors of $w$. Similarly we can compute degeneracy homomorphism $s_{i}^{G^{\prime} *_{G} G^{\prime \prime}}$ on $\left(G^{\prime} *_{G} G^{\prime \prime}\right)_{n}$ in the same manner. 
There is a classifying space functor from the category of simplicial groups to the category of simplicial sets, denoted by $\bar{W}$, with the property that the geometric realization of $\bar{W}(G)$ is a classifying space of the geometric realization of the simplicial group $G$. We refer to Curtis's paper [10] for the detailed construction of the functor $\bar{W}$.

An important property of free product with amalgamation on simplicial groups is that the classifying space of $G^{\prime} *_{G} G^{\prime \prime}$ can be controlled. This property is a simplicial consequence of the classical asphericity result of J H C Whitehead [21, Theorem 5] in 1939 and the formal statement of the following theorem was given in Kan and Thurston's paper [15, Proposition 4.3].

Theorem 3.2 (Whitehead Theorem) Let $\phi: G \rightarrow G^{\prime}$ and $\psi: G \rightarrow G^{\prime \prime}$ be simplicial monomorphisms. Then the classifying space $\bar{W}\left(G^{\prime} *_{G} G^{\prime \prime}\right)$ is the homotopy push-out of the diagram:

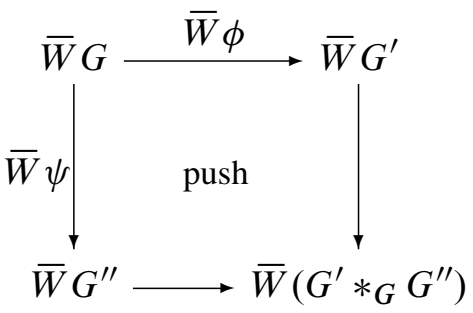

\section{Description of homotopy groups of spheres and proof of Theorem 2.2}

In this section, we are going to construct a simplicial group model $\mathcal{T}\left(S^{k}\right)$ for $\Omega S^{k}$, $k \geq 3$, by using pure braid groups. From this, we are able to give a combinatorial description of the homotopy group $\pi_{q}\left(S^{k}\right)$ for general $q$.

\subsection{Milnor's $F[K]$-construction on spheres}

Let $K$ be a simplicial set with a fixed choice of base-point $s_{0}^{n} x_{0} \in K_{n}$. Milnor [19] constructed a simplicial group $F[K]$ where $F\left[K_{n}\right]$ is the free group generated by $K_{n}$ subject to the single relation that $s_{0}^{n} x_{0}=1$. The face and degeneracy homomorphisms on $F[K]$ are induced by the face and degeneracy functions on $K$. An important property of Milnor's construction is that the geometric realization $|K|$ of $F[K]$ is homotopy equivalent to $\Omega \Sigma|K|$. (Note that in Milnor's paper [19], $K$ is required to be a reduced simplicial set. This result actually holds for any pointed simplicial set by a more general result [23, Theorem 4.9].) 
We are interested in specific simplicial group models for $\Omega S^{k+1}$ and so we start by considering the simplicial $k$-sphere $S^{k}$. Recall that the simplicial $k$-simplex $\Delta[k]$ can be defined explicitly as follows:

$\Delta[k]_{n}=\left\{\left(i_{0}, i_{1}, \ldots, i_{n}\right) \mid 0 \leq i_{0} \leq i_{1} \leq \cdots \leq i_{n} \leq k\right\}$ with $d_{i}: \Delta[k]_{n} \rightarrow \Delta[k]_{n-1}$ given by removing the $(i+1)^{\mathrm{st}}$ coordinate and $s_{i}: \Delta[k]_{n} \rightarrow \Delta[k]_{n+1}$ given by doubling the $(i+1)^{\mathrm{st}}$ coordinate for $0 \leq i \leq n$.

Let $\sigma_{k}=(0,1, \ldots, k) \in \Delta[k]_{k}$ and let $\partial \Delta[k]$ be the simplicial subset of $\Delta[k]$ generated by the faces $d_{0} \sigma_{k}, \ldots, d_{k} \sigma_{k}$. Namely $\partial \Delta[k]$ is the smallest simplicial subset of $\Delta[k]$ containing $d_{i} \sigma_{k}$ for $0 \leq i \leq k$. Let $S^{k}=\Delta[k] / \partial \Delta[k]$. Then the geometric realization $\left|S^{k}\right|$ is homeomorphic to the standard $k$-sphere $S^{k}$. As a simplicial set, $S_{n}^{k}=\{*\}$ for $n<k$ and

$$
\begin{aligned}
S_{n}^{k}=\left\{*,\left(i_{0}, i_{1}, \ldots, i_{n}\right) \mid 0 \leq i_{0} \leq i_{1} \leq \cdots \leq i_{n} \leq k\right. \\
\left.\quad \text { with }\{0,1, \ldots, k\}=\left\{i_{0}, i_{1}, \ldots, i_{n}\right\}\right\} \\
=\left\{*, s_{j_{n-k}} s_{j_{n-k-1}} \cdots s_{j_{1}} \sigma_{k} \mid 0 \leq j_{1}<j_{2}<\cdots<j_{n-k} \leq n-1\right\}
\end{aligned}
$$

for $n \geq k$. In the first description above, it is required that each $0 \leq j \leq k$ appears at least once in the sequence $\left(i_{0}, \ldots, i_{n}\right)$. In this description, we can describe the faces and degeneracies by removing-doubling coordinates where we identify the sequence $\left(i_{0}, \ldots, i_{n}\right)$ to be the base-point if any one of $0 \leq j \leq k$ does not appear in $\left(i_{0}, \ldots, i_{n}\right)$. In the second description, we can use the simplicial identities to describe the faces and degeneracies on $S^{k}$.

By applying Milnor's construction to $S^{k}$, we obtain the simplicial group $F\left[S^{k}\right] \simeq$ $\Omega S^{k+1}$ with $F\left[S^{k}\right]_{n}$ a free group of rank $\left(\begin{array}{l}n \\ k\end{array}\right)$. The generators for $F\left[S^{k}\right]_{n}$ are given in formula (4-1) with $*=1$.

\subsection{The simplicial group $\mathrm{AP}_{*}$}

There is a canonical simplicial group arising from pure braid groups systematically investigated in [3]. We are only interested in classical Artin pure braids and so we follow the discussion in [7]. Let $\mathrm{AP}_{n}=P_{n+1}$ with the face homomorphism

$$
d_{i}: \mathrm{AP}_{n}=P_{n+1} \longrightarrow \mathrm{AP}_{n-1}=P_{n}
$$

given by removing the $(i+1)^{\mathrm{st}}$ strand of $(n+1)$-strand pure braids and the degeneracy homomorphism

$$
s_{i}: \mathrm{AP}_{n}=P_{n+1} \longrightarrow \mathrm{AP}_{n+1}=P_{n+2}
$$

given by doubling the $(i+1)^{\text {st }}$ strand of $(n+1)$-strand pure braids for $0 \leq i \leq n$. Then $\mathrm{AP}_{*}$ forms a simplicial group. Let $A_{i, j}, 1 \leq i<j \leq n+1$, be the standard 
generators for $\mathrm{AP}_{n}=P_{n+1}$. Then the face operations in the simplicial group $\mathrm{AP}_{*}$ are defined as follows:

$$
d_{t}\left(A_{i, j}\right)= \begin{cases}A_{i-1, j-1} & \text { if } t+1<i, \\ 1 & \text { if } t+1=i, \\ A_{i, j-1} & \text { if } i<t+1<j, \\ 1 & \text { if } t+1=j, \\ A_{i, j} & \text { if } t+1>j .\end{cases}
$$

And the degeneracy operations are defined as follows:

$$
s_{t}\left(A_{i, j}\right)= \begin{cases}A_{i+1, j+1} & \text { if } t+1<i, \\ A_{i, j+1} \cdot A_{i+1, j+1} & \text { if } t+1=i, \\ A_{i, j+1} & \text { if } i<t+1<j, \\ A_{i, j} \cdot A_{i, j+1} & \text { if } t+1=j, \\ A_{i, j} & \text { if } t+1>j .\end{cases}
$$

Observe that $\mathrm{AP}_{1}=P_{2} \cong \mathbb{Z}$ is generated by $A_{1,2}$ with $d_{0} A_{1,2}=d_{1} A_{1,2}=1$. The representing simplicial map

$$
f_{A_{1,2}}: S^{1} \longrightarrow \mathrm{AP}_{*}
$$

with $f_{\sigma_{1}}=A_{1,2}$ extends uniquely to a simplicial homomorphism

$$
\Theta: F\left[S^{1}\right] \longrightarrow \mathrm{AP}_{*} .
$$

The following embedding theorem plays an important role for our constructions of simplicial group models for the loop spaces of spheres and Moore spaces.

Theorem 4.1 [7, Theorem 1.2] The simplicial homomorphism

$$
\Theta: F\left[S^{1}\right] \longrightarrow \mathrm{AP}_{*}
$$

is a simplicial monomorphism.

\subsection{Simplicial group models for $\Omega S^{k}$ with $k \geq 3$}

Assume that $k \geq 3$. Let $\alpha \in F\left[S^{1}\right]_{k-2}$ such that

(1) $\alpha \neq 1$ and

(2) $d_{j} \alpha=1$ for all $0 \leq j \leq k-2$, that is, $\alpha$ is a Moore cycle.

(Note: We do not assume that $\alpha$ induces a nontrivial element in $\pi_{k-2}\left(F\left[S^{1}\right]\right)=$ $\pi_{k-1}\left(S^{2}\right)$. There are many choices for such an $\alpha$. We will give a particular choice of 
$\alpha$ with braided instructions later. For a moment $\alpha$ is given by any nontrivial Moore cycle.) The representing simplicial map

$$
f_{\alpha}: S^{k-2} \longrightarrow F\left[S^{1}\right]
$$

extends uniquely to a simplicial homomorphism

$$
\tilde{f}_{\alpha}: F\left[S^{k-2}\right] \longrightarrow F\left[S^{1}\right]
$$

by the universal property of Milnor's construction.

Lemma 4.2 Let $k \geq 3$ and let $\alpha \neq 1 \in F\left[S^{1}\right]_{k-2}$ be a Moore cycle. Then the map

$$
\tilde{f}_{\alpha}: F\left[S^{k-2}\right] \longrightarrow F\left[S^{1}\right]
$$

is a simplicial monomorphism.

Proof Let $G=\tilde{f}_{\alpha}\left(F\left[S^{k-2}\right]\right)$ be the image of $\tilde{f}_{\alpha}$. Then $G$ is a simplicial subgroup of $F\left[S^{1}\right]$. Since $F\left[S^{1}\right]_{q}$ is a free group, $G_{q}$ is free group for each $q$. The statement will follow if we can prove that the simplicial epimorphism

$$
\tilde{f}_{\alpha}: F\left[S^{k-2}\right] \longrightarrow G
$$

is a simplicial monomorphism. Observe that since each $F\left[S^{k-2}\right]_{q}$ is a free group, which is residually nilpotent, it suffices to show that the morphism of the associated Lie algebras induced from the lower central series

$$
L\left(\tilde{f}_{\alpha}\right): L\left(F\left[S^{k-1}\right]\right) \longrightarrow L(G)
$$

is a simplicial isomorphism. For each $q$, since both $F\left[S^{k-2}\right]_{q}$ and $G_{q}$ are free groups, their associated Lie algebras are the free Lie algebras generated by their Abelianizations. Thus it suffices to show that

$$
\tilde{f}_{\alpha}^{\mathrm{ab}}: F\left[S^{k-2}\right]^{\mathrm{ab}}=K(\mathbb{Z}, k-2) \longrightarrow G^{\mathrm{ab}}
$$

is a simplicial isomorphism.

Note that the Moore chain complex of $K(\mathbb{Z}, k-2)$ is given by

$$
N_{q} K(\mathbb{Z}, k-2)= \begin{cases}0 & \text { if } q \neq k-2 \\ \mathbb{Z} & \text { if } q=k-2\end{cases}
$$

Since $\tilde{f}_{\alpha}^{\mathrm{ab}}: K(\mathbb{Z}, k-2) \rightarrow G^{\mathrm{ab}}$ is a simplicial epimorphism,

$$
N\left(\tilde{f}_{\alpha}^{\mathrm{ab}}\right): N_{q} K(\mathbb{Z}, k-2) \longrightarrow N_{q} G^{\mathrm{ab}}
$$


is an epimorphism for any $q$ by Proposition 3.1. It follows that $N_{q} G^{\mathrm{ab}}=0$ for $q \neq k-2$. For $q=k-2$, we have $N_{k-2} G^{\mathrm{ab}}=G_{k-2}=\langle\alpha\rangle \cong \mathbb{Z}$ with

$$
N\left(\tilde{f}_{\alpha}^{\mathrm{ab}}\right): N_{k-2} K(\mathbb{Z}, k-2) \cong \mathbb{Z} \longrightarrow N_{k-2} G^{\mathrm{ab}} \cong \mathbb{Z}
$$

an isomorphism from the definition of $f_{\alpha}$. Thus

$$
N\left(\tilde{f}_{\alpha}^{\mathrm{ab}}\right): N F\left[S^{k-2}\right]^{\mathrm{ab}} \longrightarrow N G^{\mathrm{ab}}
$$

is an isomorphism. By Proposition 3.1, $\tilde{f}_{\alpha}^{\mathrm{ab}}: F\left[S^{k-2}\right]^{\mathrm{ab}}=K(\mathbb{Z}, k-2) \longrightarrow G^{\mathrm{ab}}$ is a simplicial isomorphism. This finishes the proof.

Now, by Theorem 4.1 and Lemma 4.2, the composite

$$
\phi_{\alpha}: F\left[S^{k-2}\right] \stackrel{\tilde{f}_{\alpha}}{\longrightarrow} F\left[S^{1}\right] \stackrel{\Theta}{\longrightarrow} \mathrm{AP}_{*}
$$

is a simplicial monomorphism. Define the simplicial group $\mathcal{T}\left(S^{k} ; \alpha\right)$ to be the free product with amalgamation defined by the diagram

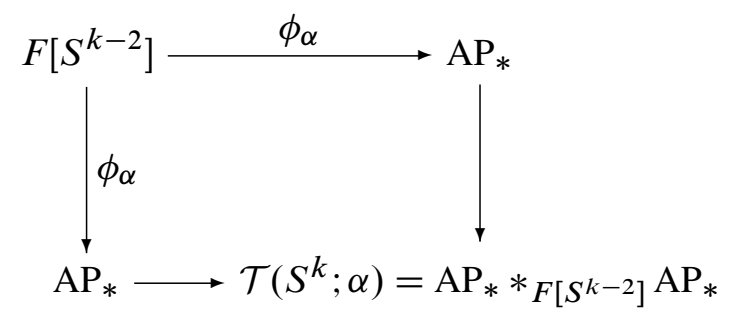

Theorem 4.3 Let $k \geq 3$ and let $\alpha \neq 1 \in F\left[S^{1}\right]_{k-2}$ be a Moore cycle. Then the geometric realization of the simplicial group $\mathcal{T}\left(S^{k} ; \alpha\right)$ is homotopy equivalent to $\Omega S^{k}$.

Proof By Theorem 3.2, the classifying space $\bar{W} \mathcal{T}\left(S^{k} ; \alpha\right)$ is the homotopy push-out of

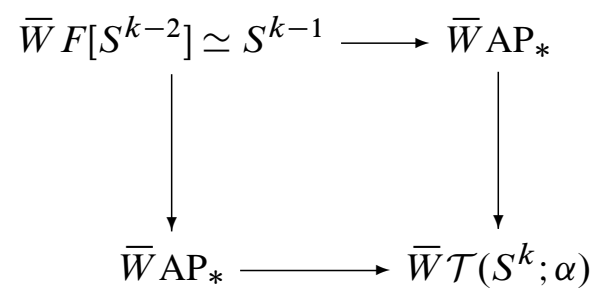

$\mathrm{By}$ [7, Theorem 1.1] (the complete proof is given in [8]), $\mathrm{AP}_{*}$ is a contractible simplicial group and so $\bar{W} \mathrm{AP}_{*}$ is contractible. It follows that

$$
\bar{W} \mathcal{T}\left(S^{k} ; \alpha\right) \simeq S^{k}
$$

and hence the result. 


\subsection{Some technical lemmas}

Recall (see Magnus, Karrass and Solitar [17, p. 288-289]) that a bracket arrangement of weight $n$ in a group $G$ is a map $\beta^{n}: G^{n} \rightarrow G$, which is defined inductively as follows:

$$
\beta^{1}=\operatorname{id}_{G}, \quad \beta^{2}\left(a_{1}, a_{2}\right)=\left[a_{1}, a_{2}\right]
$$

for any $a_{1}, a_{2} \in G$. Suppose that the bracket arrangements of weight $k$ are defined for $1 \leq k<n$ with $n \geq 3$. A map $\beta^{n}: G^{n} \rightarrow G$ is called a bracket arrangement of weight $n$ if $\beta^{n}$ is the composite

$$
G^{n}=G^{k} \times G^{n-k} \stackrel{\beta^{k} \times \beta^{n-k}}{\longrightarrow} G \times G \stackrel{\beta^{2}}{\longrightarrow} G
$$

for some bracket arrangements $\beta^{k}$ and $\beta^{n-k}$ of weight $k$ and $n-k$, respectively, with $1 \leq k<n$. For instance, if $n=3$, there are two bracket arrangements given by $\left[\left[a_{1}, a_{2}\right], a_{3}\right]$ and $\left[a_{1},\left[a_{2}, a_{3}\right]\right]$.

Let $R_{j}$ be a sequence of subgroups of $G$ for $1 \leq j \leq n$. The fat commutator subgroup $\left[\left[R_{1}, R_{2}, \ldots, R_{n}\right]\right]$ is defined to be the subgroup of $G$ generated by all of the commutators

$$
\beta^{t}\left(g_{i_{1}}, \ldots, g_{i_{t}}\right)
$$

where

(1) $1 \leq i_{s} \leq n$,

(2) $\left\{i_{1}, \ldots, i_{t}\right\}=\{1, \ldots, n\}$, that is each integer in $\{1,2, \cdots, n\}$ appears as at least one of the integers $i_{s}$,

(3) $g_{j} \in R_{j}$,

(4) $\beta^{t}$ runs over all of the bracket arrangements of weight $t$ (with $t \geq n$ ).

For convenience, let $\left[\left[R_{1}\right]\right]=R_{1}$.

The symmetric commutator subgroup $\left[R_{1}, R_{2}, \ldots, R_{n}\right]_{S}$ defined by

$$
\left[R_{1}, R_{2}, \ldots, R_{n}\right]_{S}=\prod_{\sigma \in \Sigma_{n}}\left[\ldots\left[R_{\sigma(1)}, R_{\sigma(2)}\right], \ldots, R_{\sigma(n)}\right],
$$

where $\left[\ldots\left[R_{\sigma(1)}, R_{\sigma(2)}\right], \ldots, R_{\sigma(n)}\right]$ is the subgroup generated by the left iterated commutators

$$
\left[\ldots\left[\left[g_{1}, g_{2}\right], g_{3}\right], \ldots, g_{n}\right]
$$

with $g_{i} \in R_{\sigma(i)}$. For convenience, let $\left[R_{1}\right]_{S}=R_{1}$. From the definition, the symmetric commutator subgroup is a subgroup of the fat commutator subgroup. In fact they are the same subgroup by the following theorem, provided that each $R_{j}$ is normal. 
Lemma 4.4 [16, Theorem 1.1] Let $R_{j}$ be any normal subgroup of a group $G$ with $1 \leq j \leq n$. Then

$$
\left[\left[R_{1}, R_{2}, \ldots, R_{n}\right]\right]=\left[R_{1}, R_{2}, \ldots, R_{n}\right]_{S}
$$

One can determine the Moore chains and boundaries for the self free products of $\mathrm{AP}_{*}$ with a help of the Kurosh theorem on the structure of subgroups of free products. However, in order to get this description, we will use another method. We construct a simplicial free group $\mathcal{G}$ as follows: For each $n \geq 0$, the group $\mathcal{G}_{n}$ is the free group generated by $x_{i, j}$ for $1 \leq i<j \leq n+1$. The face and degeneracy operations are given by formulae (4-2) and (4-3), where we replace $A_{i, j}$ by $x_{i, j}$. It is straightforward to check that the simplicial identities hold. Thus we have a simplicial group $\mathcal{G}$.

Now we are going to determine the Moore chains and Moore cycles of the free products of $\mathcal{G}$. Let $J$ be an index set and let $\mathcal{G}^{* J}=*_{\alpha \in J} \mathcal{G}(\alpha)$, where each $\mathcal{G}(\alpha)$ is a copy of $\mathcal{G}$ indexed by an element $\alpha \in J$. For each group $\mathcal{G}(\alpha)_{n}=\mathcal{G}_{n}$, let $x_{i, j}(\alpha)$ denote the generator $x_{i, j}$ for $1 \leq i<j \leq n+1$. From the definition, $\mathcal{G}_{n}^{* J}=*_{\alpha \in J} \mathcal{G}(\alpha)_{n}$ is a free group with a basis given by $\left\{x_{i, j}(\alpha) \mid 1 \leq i<j \leq n+1, \alpha \in J\right\}$.

A basic word in the group $\mathcal{G}_{n}^{* J}$ means one of the elements $x_{i, j}(\alpha)^{ \pm 1}$ for some $\alpha \in J$ and so $1 \leq i<j \leq n+1$. Let

$$
w=\beta_{t}\left(x_{i_{1}, j_{1}}\left(\alpha_{1}\right)^{ \pm 1}, x_{i_{2}, j_{2}}\left(\alpha_{2}\right)^{ \pm 1}, \ldots, x_{i_{t}, j_{t}}\left(\alpha_{t}\right)^{ \pm 1}\right)
$$

be a $t$-fold iterated commutator on basic words, where the bracket $\beta_{t}(\cdots)$ is any bracket arrangement. Define

$$
\operatorname{Index}(w)=\left\{i_{1}, j_{1}, i_{2}, j_{2}, \ldots, i_{t}, j_{t}\right\} \subseteq\{1,2, \ldots, n+1\} .
$$

(Note: In our definition, $\operatorname{Index}(w)$ is only well-defined for commutators with entries from basic words.)

For each pair $1 \leq i<j \leq n+1$, let

$$
R_{i, j}^{J}=\left\langle x_{i, j}(\alpha) \mid \alpha \in J\right\rangle^{\mathcal{G}^{* J}}
$$

be the normal closure of the elements $x_{i, j}(\alpha), \alpha \in J$, in the group $\mathcal{G}^{* J}$. For a subset $T \subseteq\{1,2, \ldots, n+1\}$, define

$$
R[T]=\prod_{T \subseteq\left\{i_{1}, j_{1}, i_{2}, j_{2}, \ldots, i_{t}, j_{t}\right\}}\left[\ldots\left[R_{i_{1}, j_{1}}, R_{i_{2}, j_{2}}\right], \ldots, R_{i_{t}, j_{t}}\right]
$$

to be the product of the iterated commutator subgroup of $R_{i, j}$ such that each number in $T$ occurs at least once in the indices of $R_{i, j}$. (Here if $t=1$, then we let commutator 
subgroup $\left[R_{i_{1}, j_{1}}\right]=R_{i_{1}, j_{1}}$ by convention.) In the case that $T=\{1,2, \ldots, n+1\}$, we denote

$$
\left[R_{i, j} \mid 1 \leq i<j \leq n+1\right]_{S}
$$

by $R[1,2, \ldots, n+1]$.

Lemma 4.5 Let $\mathcal{G}^{* J}$ be the self free product of $\mathcal{G}$ over a set $J$. Then:

(1) The Moore chains are $N_{n} \mathcal{G}^{* J}=R[2,3, \ldots, n+1]$.

(2) The Moore cycles are $\mathcal{Z}_{n} \mathcal{G}^{* J}=R[1,2,3, \ldots, n+1]=\left[R_{i, j} \mid 1 \leq i<j \leq n+1\right]_{S}$.

(3) The Moore boundaries are

$$
\mathcal{B}_{n} \mathcal{G}^{* J}=\mathcal{Z}_{n} \mathcal{G}^{* J}=R[1,2,3, \ldots, n+1]=\left[R_{i, j} \mid 1 \leq i<j \leq n+1\right]_{S} .
$$

Proof For assertions (1) and (2), the direction

$$
R[2,3, \ldots, n+1] \leq N_{n} \mathcal{G}^{* J} \text { and } R[1,2,3, \ldots, n+1] \leq \mathcal{Z}_{n} \mathcal{G}^{* J}
$$

can be easily checked as follows. From equation (4-2), we have $d_{k} x_{i, j}(\alpha)=1$ for $\alpha \in J$ if $k+1=i$ or $j$. Thus

$$
R_{i, j} \leq \operatorname{Ker}\left(d_{k}: \mathcal{G}_{n}^{* J} \rightarrow \mathcal{G}_{n-1}^{* J}\right)
$$

if $k+1=i$ or $j$. Thus

$$
\left[\ldots\left[R_{i_{1}, j_{1}}, R_{i_{2}, j_{2}}\right], \ldots, R_{i_{t}, j_{t}}\right] \leq N_{n} \mathcal{G}^{* J}=\bigcap_{k=1}^{n} \operatorname{Ker}\left(d_{k}: \mathcal{G}_{n}^{* J} \rightarrow \mathcal{G}_{n-1}^{* J}\right)
$$

if $\{2,3, \ldots, n+1\} \subseteq\left\{i_{1}, j_{1}, i_{2}, j_{2}, \ldots, i_{t}, j_{t}\right\}$ since each $d_{k}, 1 \leq k \leq n$, sends one of entries $R_{i_{s}, j_{s}}$ in this (iterated) commutator subgroup to the trivial group. It follows that $R[2,3, \ldots, n+1] \leq N_{n} \mathcal{G}^{* J}$. Similarly $R[1,2, \ldots, n+1] \leq \mathcal{Z}_{n+1} \mathcal{G}^{* J}$. Thus the main point is to prove that

$$
N_{n} \mathcal{G}^{* J} \leq R[2,3, \ldots, n+1] \quad \text { and } \quad \mathcal{Z}_{n} \mathcal{G}^{* J} \leq R[1,2, \ldots, n+1] .
$$

If $n=1$, then $R[2]=R[1,2]=\mathcal{G}_{1}^{* J}$ because $\mathcal{G}_{1}^{* J}$ is generated by $x_{1,2}(\alpha)$ for $\alpha \in J$. In this case, the identity that $N_{1} \mathcal{G}^{* J}=\mathcal{Z}_{1} \mathcal{G}^{* J}=R[2]=R[1,2]=\mathcal{G}_{1}^{* J}$ holds. Thus we may assume that $n \geq 2$.

We first consider the last face operation

$$
d_{n}: \mathcal{G}_{n}^{* J} \longrightarrow \mathcal{G}_{n-1}^{* J} .
$$


Let $K_{n}=\operatorname{Ker}\left(d_{n}: \mathcal{G}_{n}^{* J} \rightarrow \mathcal{G}_{n-1}^{* J}\right)$. From equation (4-2),

$$
d_{n}\left(x_{i, j}(\alpha)\right)= \begin{cases}1 & \text { if } 1 \leq i<j=n+1, \\ x_{i, j}(\alpha) & \text { if } 1 \leq i<j \leq n\end{cases}
$$

Observe that the basis of $\mathcal{G}_{n}^{* J}$ is given by the disjoint union of the basis of $\mathcal{G}_{n-1}^{* J}$ with the set $\left\{x_{i, n+1}(\alpha) \mid 1 \leq i<n+1, \alpha \in J\right\}$. By [24, Proposition 3.3], a basis for the free group $K_{n}$ is given by the subset $X_{n}$ of $\mathcal{G}_{n}^{* J}$ consisting of all of the following iterated commutators on basic words

$$
w=\left[\ldots\left[\left[x_{i, n}\left(\alpha_{0}\right), x_{i_{1}, j_{1}}^{\epsilon_{1}}\left(\alpha_{1}\right)\right], x_{i_{2}, j_{2}}^{\epsilon_{2}}\left(\alpha_{2}\right)\right], \ldots, x_{i_{t}, j_{t}}^{\epsilon_{t}}\left(\alpha_{t}\right)\right],
$$

where

(1) $t \geq 0$ (Here if $t=0$, then $w=\left[x_{i, n}\left(\alpha_{0}\right)\right]=x_{i, n}\left(\alpha_{0}\right)$.)

(2) $\epsilon_{s}= \pm 1$ for $1 \leq s \leq t$,

(3) $1 \leq i_{s}<j_{s} \leq n$ for $1 \leq s \leq t$,

(4) $\alpha_{s} \in J$ for $0 \leq s \leq t$ and

(5) the word $x_{i_{1}, j_{1}}^{\epsilon_{1}}\left(\alpha_{1}\right) x_{i_{2}, j_{2}}^{\epsilon_{2}}\left(\alpha_{2}\right) \cdots x_{i_{t}, j_{t}}^{\epsilon_{t}}\left(\alpha_{t}\right)$ is an irreducible word in the group $\mathcal{G}_{n-1}^{* J} \leq \mathcal{G}_{n}^{* J}$.

Next we consider the face operation $d_{k}$ restricted to $K_{n}$ for $0 \leq k<n$. From the $\Delta$-identity $d_{k} d_{n}=d_{n-1} d_{k}$ for $1 \leq k \leq n-1$, we have the commutative diagram of short exact sequence of groups

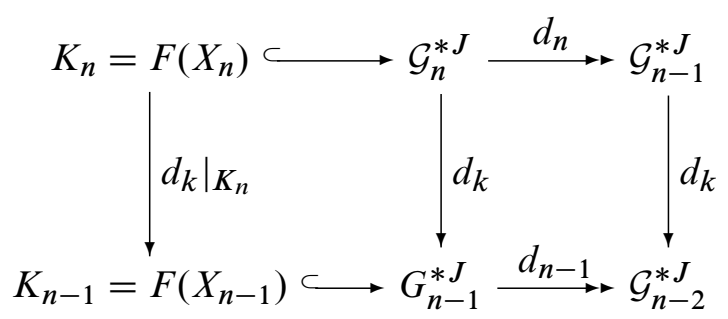

for $1 \leq k \leq n-1$. Consider $d_{k} w$ for $w \in X_{n}$. From equation (4-2), $d_{k} x_{i, n}(\alpha)$ is given by the following table:

$$
d_{k}\left(\begin{array}{ccccccc}
x_{1, n}(\alpha) & \cdots & x_{k-1, n}(\alpha) & x_{k, n}(\alpha) & x_{k+1, n}(\alpha) & \cdots & x_{n-1, n}(\alpha) \\
\downarrow & & \downarrow & \downarrow & \downarrow & & \downarrow \\
x_{1, n-1}(\alpha) & \cdots & x_{k-1, n}(\alpha) & 1 & x_{k, n-1}(\alpha) & \cdots & x_{n-2, n-1}(\alpha)
\end{array}\right)
$$

We now start to prove statement (4-4). Let

$$
X_{n}(k)=\left\{w \in X_{n} \mid k+1 \in \operatorname{Index}(w)\right\}
$$


for $0 \leq k \leq n-1$. If $w \in X_{n}(k)$, then $d_{k} w=1$ as $d_{k}$ sends one of the entries in the commutator $w$ to 1 . Let $w \in X_{n} \backslash X_{n}(k)$ be written as in (4-5). Then $i \neq k+1$ and $k+1 \notin\left\{i_{1}, j_{1}, \ldots, i_{t}, j_{t}\right\}$. From the above table, $d_{k} x_{i, n}\left(\alpha_{0}\right)=x_{i, n-1}\left(\alpha_{0}\right)$ for $i<k+1$ and $x_{i-1, n-1}\left(\alpha_{0}\right)$ for $i>k+1$. For other entries $x_{i_{s}, j_{s}}^{\epsilon_{s}}\left(\alpha_{s}\right)$, we have

$$
d_{k}\left(x_{i_{s}, j_{s}}^{\epsilon_{s}}\left(\alpha_{s}\right)\right)= \begin{cases}x_{i_{s}-1, j_{s}-1}^{\epsilon_{s}}\left(\alpha_{s}\right) & \text { if } k+1<i_{s}, \\ x_{i_{s}}^{\epsilon_{s}, j_{s}-1}\left(\alpha_{s}\right) & \text { if } i_{s}<k+1<j_{s}, \\ x_{i_{s}, j_{s}}^{\epsilon_{s}}\left(\alpha_{s}\right) & \text { if } k+1>j_{s} .\end{cases}
$$

Observe that

$d_{k}:\left\{x_{i, j}(\alpha) \mid \alpha \in J, 1 \leq i<j \leq n\right.$ and $\left.k+1 \neq i, j\right\} \rightarrow\left\{x_{i, j}(\alpha) \mid \alpha \in J, 1 \leq i<j \leq n-1\right\}$

is a bijection. The restriction of $d_{k}$ in the subgroup

$$
d_{k} \mid: F\left(x_{i, j}(\alpha) \mid \alpha \in J, 1 \leq i<j \leq n \text { and } k+1 \neq i, j\right) \longrightarrow \mathcal{G}_{n-2}^{* J}
$$

is an isomorphism. Since the word

$x_{i_{1}, j_{1}}^{\epsilon_{1}}\left(\alpha_{1}\right) x_{i_{2}, j_{2}}^{\epsilon_{2}}\left(\alpha_{2}\right) \cdots x_{i_{t}, j_{t}}^{\epsilon_{t}}\left(\alpha_{t}\right) \in F\left(x_{i, j}(\alpha) \mid \alpha \in J, 1 \leq i<j \leq n\right.$ and $\left.k+1 \neq i, j\right)$

is irreducible, the word

$$
\begin{aligned}
d_{k}\left(x_{i_{1}, j_{1}}^{\epsilon_{1}}\left(\alpha_{1}\right) x_{i_{2}, j_{2}}^{\epsilon_{2}}\left(\alpha_{2}\right) \cdots x_{i_{t}, j_{t}}^{\epsilon_{t}}\left(\alpha_{t}\right)\right) & \\
& =\left(d_{k} x_{i_{1}, j_{1}}\right)^{\epsilon_{1}}\left(\alpha_{1}\right)\left(d_{k} x_{i_{2}, j_{2}}\right)^{\epsilon_{2}}\left(\alpha_{2}\right) \cdots\left(d_{k} x_{i_{t}, j_{t}}\right)^{\epsilon_{t}}\left(\alpha_{t}\right)
\end{aligned}
$$

is irreducible in $\mathcal{G}_{n-2}^{* J} \leq \mathcal{G}_{n-1}^{* J}$. It follows that $d_{k} w \in X_{n-1}$ for each $w \in X_{n} \backslash X_{n}(k)$ and the function

$$
d_{k}: X_{n} \backslash X_{n}(k) \longrightarrow X_{n-1}
$$

is a bijection. This allows us to apply the algorithm in [24, Section 3] to

$$
d_{k} \mid: K_{n}=F\left(X_{n}\right) \longrightarrow K_{n-1}=F\left(X_{n-1}\right)
$$

for $0 \leq k \leq n-1$ in diagram (4-6) and so, by [24, Theorem 3.4], the Moore chains

$$
N_{n} \mathcal{G}^{* J}=\bigcap_{k=1}^{n-1} \operatorname{Ker}\left(d_{k} \mid: K_{n} \rightarrow K_{n-1}\right)
$$

are generated by certain iterated commutators

$$
w=\beta_{t}\left(x_{i_{1}, j_{1}}\left(\alpha_{1}\right)^{ \pm 1}, x_{i_{2}, j_{2}}\left(\alpha_{2}\right)^{ \pm 1}, \ldots, x_{i_{t}, j_{t}}\left(\alpha_{t}\right)^{ \pm 1}\right)
$$


with $\{2,3, \ldots, n+1\} \in \operatorname{Index}(w)$ and the Moore cycles

$$
\mathcal{Z}_{n} \mathcal{G}^{* J}=\bigcap_{k=0}^{n-1} \operatorname{Ker}\left(d_{k} \mid: K_{n} \rightarrow K_{n-1}\right)
$$

are generated by certain iterated commutators

$$
w=\beta_{t}\left(x_{i_{1}, j_{1}}\left(\alpha_{1}\right)^{ \pm 1}, x_{i_{2}, j_{2}}\left(\alpha_{2}\right)^{ \pm 1}, \ldots, x_{i_{t}, j_{t}}\left(\alpha_{t}\right)^{ \pm 1}\right)
$$

with $\{1,2, \ldots, n+1\} \in \operatorname{Index}(w)$. (Note: The commutator $w$ in (4-7) or (4-8) may not be in the standard form from left to right.) Since each entry $x_{i_{s}, j_{s}}\left(\alpha_{s}\right)^{ \pm 1}$ belongs to $R_{i_{s}}, j_{s}$, the commutator $w$ in (4-7) or (4-8) lies in the fat commutator subgroup $\left[\left[R_{i_{1}, j_{1}}, R_{i_{2}, j_{2}}, \ldots, R_{i_{t}, j_{t}}\right]\right]$ and so, by Lemma 4.4 ,

$$
w \in \prod_{\sigma \in \Sigma_{t}}\left[\ldots\left[R_{i_{\sigma(1)}, j_{\sigma(1)}}, R_{i_{\sigma(2)}, j_{\sigma(2)}}\right], \ldots, R_{i_{\sigma(t)}, j_{\sigma(t)}}\right] \leq R[s, s+1, \ldots, n+1],
$$

where $s=2$ in the case of (4-7) and $s=1$ in the case of (4-8). This finishes the proof of statement (4-4) and hence assertions (1) and (2).

(3) By assertion (2),

$$
\mathcal{Z}_{n} \mathcal{G}^{* J}=\prod_{\{1,2, \ldots, n+1\} \subseteq\left\{i_{1}, j_{1}, i_{2}, j_{2}, \ldots, i_{t}, j_{t}\right\}}\left[\ldots\left[R_{i_{1}, j_{1}}, R_{i_{2}, j_{2}}\right], \ldots, R_{i_{t}, j_{t}}\right] .
$$

From equation (4-2), we have $d_{0} x_{i+1, j+1}(\alpha)=x_{i, j}(\alpha)$ for $1 \leq i<j \leq n+1$ and $\alpha \in J$. Thus

$$
d_{0}\left(R_{i+1, j+1}\right)=R_{i, j}
$$

for $1 \leq i<j \leq n+1$. Given a factor $\left[\ldots\left[R_{i_{1}, j_{1}}, R_{i_{2}, j_{2}}\right], \ldots, R_{i_{t}, j_{t}}\right]$ in $\mathcal{Z}_{n} \mathcal{G}^{* J}$ with $\{1,2, \ldots, n+1\} \subseteq\left\{i_{1}, j_{1}, i_{2}, j_{2}, \ldots, i_{t}, j_{t}\right\}$, we have

$d_{0}\left(\left[\ldots\left[R_{i_{1}+1, j_{1}+1}, R_{i_{2}+1, j_{2}+1}\right], \ldots, R_{i_{t}+1, j_{t}+1}\right]\right)$

$$
=\left[\ldots\left[R_{i_{1}, j_{1}}, R_{i_{2}, j_{2}}\right], \ldots, R_{i_{t}, j_{t}}\right] .
$$

Since $\{2,3, \ldots, n+2\} \subseteq\left\{i_{1}+1, j_{1}+1, i_{2}+1, j_{2}+1, \ldots, i_{t}+1, j_{t}+1\right\}$, the subgroup

$$
\prod_{\{1,2, \ldots, n+1\} \subseteq}\left[\ldots\left[R_{i_{1}+1, j_{1}+1}, R_{i_{2}+1, j_{2}+1}\right], \ldots, R_{i_{t}+1, j_{t}+1}\right] \leq N_{n+1} \mathcal{G}^{* J}
$$$$
\left\{i_{1}, j_{1}, i_{2}, j_{2}, \ldots, i_{t}, j_{t}\right\}
$$

with

$$
d_{0}\left(\prod_{\substack{\{1,2, \ldots, n+1\} \subseteq \\\left\{i_{1}, j_{1}, i_{2}, j_{2}, \ldots, i_{t}, j_{t}\right\}}}\left[\ldots\left[R_{i_{1}+1, j_{1}+1}, R_{i_{2}+1, j_{2}+1}\right], \ldots, R_{i_{t}+1, j_{t}+1}\right]\right)=\mathcal{Z}_{n} \mathcal{G}^{* J} .
$$


It follows that $\mathcal{Z}_{n} \mathcal{G}^{* J} \leq \mathcal{B}_{n} \mathcal{G}^{* J}$. Assertion (3) follows and this finishes the proof.

The following lemma states that $R[1,2, \ldots, n+1]$ can be given by the product of a finite collection of commutator subgroups.

Lemma 4.6 The subgroup $R[1,2, \ldots, n+1]$ of $\mathcal{G}_{n}^{* J}$ is the product of the following commutator subgroups

$$
\left[\ldots\left[R_{i_{1}, j_{1}}, R_{i_{2}, j_{2}}\right], \ldots, R_{i_{t}, j_{t}}\right]
$$

where

(1) $\left\{i_{1}, j_{1}, i_{2}, j_{2}, \ldots, i_{t}, j_{t}\right\}=\{1,2, \ldots, n+1\}$ and

(2) $\left\{i_{1}, j_{1}, i_{2}, j_{2}, \ldots, i_{t}, j_{t}\right\} \backslash\left\{i_{p}, j_{p}\right\} \neq\{1,2, \ldots, n+1\}$ for any $1 \leq p \leq t$.

Proof Let $H$ be the product of the commutator subgroups given in the statement. Clearly $H \leq R[1,2, \ldots, n+1]$. Now consider the factor

$$
\left[\ldots\left[R_{i_{1}, j_{1}}, R_{i_{2}, j_{2}}\right], \ldots, R_{i_{t}, j_{t}}\right]
$$

with $\{1,2, \ldots, n+1\}=\left\{i_{1}, j_{1}, i_{2}, j_{2}, \ldots, i_{t}, j_{t}\right\}$ in $R[1,2, \ldots, n+1]$. If there exists $1 \leq p \leq t$ such that

$$
\left\{i_{1}, j_{1}, i_{2}, j_{2}, \ldots, i_{t}, j_{t}\right\} \backslash\left\{i_{p}, j_{p}\right\}=\{1,2, \ldots, n+1\},
$$

since $\left[\ldots\left[R_{i_{1}, j_{1}}, R_{i_{2}, j_{2}}\right], \ldots, R_{i_{p-1}, j_{p-1}}\right]$ is normal, we have

$$
\left[\left[\ldots\left[R_{i_{1}, j_{1}}, R_{i_{2}, j_{2}}\right], \ldots, R_{i_{p-1}, j_{p-1}}\right], R_{i_{p}, j_{p}}\right] \leq\left[\ldots\left[R_{i_{1}, j_{1}}, R_{i_{2}, j_{2}}\right], \ldots, R_{i_{p-1}, j_{p-1}}\right] .
$$

(If $p=1$, then we use $\left[R_{i_{1}, j_{1}}, R_{i_{2}, j_{2}}\right] \leq R_{i_{2}, j_{2}}$.) It follows that

$$
\left[\ldots\left[R_{i_{1}, j_{1}}, R_{i_{2}, j_{2}}\right], \ldots, R_{i_{t}, j_{t}}\right] \leq\left[\ldots\left[R_{i_{1}, j_{1}}, R_{i_{2}, j_{2}}\right], \ldots, \widehat{R}_{i_{p}, j_{p}}, \ldots, R_{i_{t}, j_{t}}\right]
$$

with $\left\{i_{1}, j_{1}, i_{2}, j_{2}, \ldots, i_{t}, j_{t}\right\} \backslash\left\{i_{p}, j_{p}\right\}=\{1,2, \ldots, n+1\}$. Consider the factor $\left[\ldots\left[R_{i_{1}, j_{1}}, R_{i_{2}, j_{2}}\right], \ldots, \hat{R}_{i_{p}, j_{p}}, \ldots, R_{i_{t}, j_{t}}\right]$. By repeating the above process for removing surplus entries, we have

$$
\left[\ldots\left[R_{i_{1}, j_{1}}, R_{i_{2}, j_{2}}\right], \ldots, R_{i_{t}, j_{t}}\right] \leq H
$$

and hence the result.

The following simple result is well-known and follows from the structure of normal forms of free products with amalgamation (for the proof see, for example, [12]):

Lemma 4.7 Let $G=G_{1} *_{A} G_{2}$ be a free product with amalgamation such that $G_{1} \neq A$ and $G_{2} \neq A$. Then $Z(G) \leq Z(A)$. 


\subsection{Proof of Theorem 2.2}

We use our simplicial group model $\mathcal{T}\left(S^{k}, \alpha\right)$ for $\Omega S^{k}$. Consider the construction of the subgroup $Q_{n, k}$ of $P_{n}$. By the definition of the simplicial group $\mathrm{AP}_{*}$, the iterated degeneracy operations on $\mathrm{AP}_{1}=P_{2}$ are given by the cabling and so the elements $x_{1}, \ldots, x_{k-2}$ in Step 1 are the canonical basis for the subgroup

$$
\Theta\left(F\left[S^{1}\right]_{k-2}\right) \leq \mathrm{AP}_{k-2}=P_{k-1} .
$$

Since $d_{i} x_{i}=d_{i} x_{i+1}$ for $1 \leq i \leq k-3$ and $d_{0} x_{1}=d_{k-2} x_{k-2}=1$, we have $d_{i} \alpha_{k}=1$ for $0 \leq i \leq k-2$. It follows that $\alpha_{k}$ is a Moore cycle in $F\left[S^{1}\right]_{k-2}$ with $\alpha_{k} \neq 1$. The elements $y_{j}, 1 \leq j \leq\left(\begin{array}{l}n-1 \\ k-2\end{array}\right)$, are standard basis for the subgroup

$$
\phi_{\alpha_{k}}\left(F\left[S^{k-2}\right]_{n-1}\right) \leq \mathrm{AP}_{n-1}=P_{n}
$$

since they are obtained by cabling on $\alpha_{k}$. It follows that

$$
P_{n} * Q_{n, k} P_{n}=\left(\mathrm{AP}_{*} * F\left[S^{k-2}\right] \mathrm{AP}_{*}\right)_{n-1}=\mathcal{T}\left(S^{k} ; \alpha_{k}\right)_{n-1} .
$$

Theorem 2.2 is a special case of the following slightly more general statement.

Theorem 4.8 Let $k \geq 3$ and let $\alpha \neq 1 \in F\left[S^{1}\right]_{k-2}$ be a Moore cycle. Then the simplicial group $\mathcal{T}\left(S^{k} ; \alpha\right) \simeq \Omega S^{k}$ has the following properties:

(1) In the group $\mathcal{T}\left(S^{k} ; \alpha\right)_{n-1}=P_{n} *_{F\left[S^{k-2}\right]_{n-1}} P_{n}$, the Moore boundaries

$$
\mathcal{B}_{n-1} \mathcal{T}\left(S^{k} ; \alpha\right)=\left[R_{i, j} \mid 1 \leq i<j \leq n\right]_{S} .
$$

(2) The homotopy group $\pi_{n}\left(S^{k}\right) \cong \pi_{n-1}\left(\Omega S^{k}\right) \cong \pi_{n-1}\left(\mathcal{T}\left(S^{k} ; \alpha\right)\right.$ ) is isomorphic to the center of the group

$$
\mathcal{T}\left(S^{k} ; \alpha\right)_{n-1} / \mathcal{B}_{n-1} \mathcal{T}\left(S^{k} ; \alpha\right)=\left(P_{n} *_{F\left[S^{k-2}\right]_{n-1}} P_{n}\right) /\left[R_{i, j} \mid 1 \leq i<j \leq n\right]_{S}
$$

for any $n$ if $k>3$ and any $n \neq 3$ if $k=3$.

Proof (1) By definition, the simplicial group $\mathcal{T}\left(S^{k} ; \alpha\right)$ is given by the free product with amalgamation $\mathrm{AP}_{*} * F\left[S^{k-2}\right] \mathrm{AP}_{*}$. Thus $\mathcal{T}\left(S^{k} ; \alpha\right)$ is a simplicial quotient group of the free product $\mathrm{AP}_{*} * \mathrm{AP}_{*}$. Let $\mathcal{G}$ be the simplicial group given in Section 4.4. Then $\mathrm{AP}_{*}$ is a simplicial quotient group of $\mathcal{G}$. It follows that there is a simplicial epimorphism

$$
g: \mathcal{G} * \mathcal{G} \longrightarrow \mathcal{T}\left(S^{k} ; \alpha\right)
$$

By Proposition 3.1,

$$
N(g)=g \mid: N_{n}(\mathcal{G} * \mathcal{G}) \longrightarrow N_{n}\left(\mathcal{T}\left(S^{k} ; \alpha\right)\right)
$$


is an epimorphism and so:

$$
\begin{aligned}
\mathcal{B}_{n-1}\left(\mathcal{T}\left(S^{k} ; \alpha\right)\right) & =d_{0}\left(N_{n}\left(\mathcal{T}\left(S^{k} ; \alpha\right)\right)\right) \\
& =d_{0}\left(g\left(N_{n}(\mathcal{G} * \mathcal{G})\right)\right) \\
& =g\left(d_{0}\left(N_{n}(\mathcal{G} * \mathcal{G})\right)\right) \\
& =g\left(\mathcal{B}_{n-1}(\mathcal{G} * \mathcal{G})\right)
\end{aligned}
$$

Assertion (1) follows from Lemma 4.5.

(2) Case I $k>3$ Since $\left.\mathcal{T}\left(S^{k} ; \alpha\right)_{q}=P_{q+1} *_{F[} S^{k-2}\right]_{q} P_{q+1}$ is a free product with amalgamation, the center

$$
Z\left(\mathcal{T}\left(S^{k} ; \alpha\right)_{q}\right) \leq Z\left(F\left[S^{k-2}\right]_{q}\right)=\{1\}
$$

for $q \geq k-1$ by Lemma 4.7. For $q=k-2$, then $Z\left(\mathcal{T}\left(S^{k} ; \alpha\right)_{k-2}\right) \leq F\left[S^{k-2}\right]_{k-2}=$ $\langle\alpha\rangle=\mathbb{Z}$ by Lemma 4.7. Since $\alpha$ is Moore cycle, $\alpha$ is a Brunnian braid in $P_{k-1}$. Recall that the center of $P_{k-1}$ is given by the full-twist braid $\Delta^{2}$ (Chow [6]) with the property that, by removing any one of the strands of $\Delta^{2}$, it becomes a generator for the center of $P_{k-1}$ and $d_{i} \Delta^{2} \neq 1$ for $k>3$. Since $\alpha$ is a Brunnian braid, any power $\alpha^{m} \notin Z\left(P_{k-1}\right)$ for $m \neq 0$. It follows that

$$
\alpha^{m} \notin Z\left(P_{k-1} * F\left[S^{k-2}\right]_{k-2} P_{k-1}\right)
$$

for $m \neq 0$. Thus $Z\left(\mathcal{T}\left(S^{k} ; \alpha\right)_{k-2}\right)=\{1\}$. For $q<k-2, \mathcal{T}\left(S^{k}, \alpha\right)_{q}$ is a free product and so $Z\left(\mathcal{T}\left(S^{k}, \alpha\right)_{q}\right)=\{1\}$, where for the low cases, $\mathcal{T}\left(S^{k} ; \alpha\right)_{1}=P_{2} * P_{2}$ is a free group of rank 2 and $\mathcal{T}\left(S^{k} ; \alpha\right)_{0}=\{1\}$. Thus the center $Z\left(\mathcal{T}\left(S^{k} ; \alpha\right)_{q}=\{1\}\right.$ for all $q \geq 0$. It follows from [24, Proposition 2.14] that

$$
\pi_{q}\left(\mathcal{T}\left(S^{k} ; \alpha\right)\right) \cong Z\left(\mathcal{T}\left(S^{k} ; \alpha\right)_{q} / \mathcal{B}_{q}\left(\mathcal{T}\left(S^{k} ; \alpha\right)\right)\right.
$$

for $q \geq 1$. This isomorphism also holds for $q=0$ because $\mathcal{T}\left(S^{k} ; \alpha\right)_{0}=\{1\}$.

Case II $k=3$ By the same arguments as above, we have $Z\left(\mathcal{T}\left(S^{3} ; \alpha\right)_{q}\right)=\{1\}$ for $q \geq 2$. By [24, Proposition 2.14], we have

$$
\pi_{q}\left(\mathcal{T}\left(S^{3} ; \alpha\right)\right) \cong Z\left(\mathcal{T}\left(S^{3} ; \alpha\right)_{q} / \mathcal{B}_{q}\left(\mathcal{T}\left(S^{3} ; \alpha\right)\right)\right.
$$

for $q \geq 3$. We only need to check that this isomorphism also holds for the cases $q=0,1$. (The case that $q=2$ is the exceptional case, which is excluded in the statement.) When $q=0$, both sides are trivial groups. Consider the case $q=1$. Note that $\mathrm{AP}_{1}=P_{2} \cong \mathbb{Z}$ is generated by $A_{12}$. Since $\alpha$ is not trivial, it is given by a nontrivial power of $A_{12}$. 
Let $\alpha=A_{12}^{m}$ for some $m \neq 0$. Then $\mathcal{T}\left(S^{3} ; \alpha\right)_{1}$ is given by the pushout diagram:

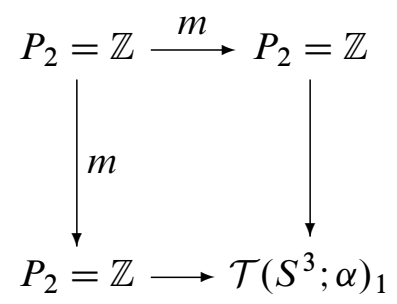

Since $R_{1,2}=\left\langle A_{1,2}, A_{1,2}^{\prime}\right\rangle^{\mathcal{T}\left(S^{3} ; \alpha\right)_{1}}=\mathcal{T}\left(S^{3}, \alpha\right)_{1}$ because $\mathcal{T}\left(S^{3} ; \alpha\right)_{1}$ is generated by $A_{1,2}$ and $A_{1,2}^{\prime}$, we have

$$
\mathcal{B}_{1}\left(\mathcal{T}\left(S^{3} ; \alpha\right)\right)=\mathcal{T}\left(S^{3} ; \alpha\right)_{1}
$$

and so

$$
Z\left(\mathcal{T}\left(S^{3} ; \alpha\right)_{1} / \mathcal{B}_{1}\left(\mathcal{T}\left(S^{3} ; \alpha\right)\right)=\mathcal{T}\left(S^{3} ; \alpha\right)_{1} / \mathcal{B}_{1}\left(\mathcal{T}\left(S^{3} ; \alpha\right)=\{1\}\right.\right.
$$

On the other hand,

$$
\pi_{1}\left(\mathcal{T}\left(S^{3} ; \alpha\right)\right)=\pi_{1}\left(\Omega S^{3}\right)=\pi_{2}\left(S^{3}\right)=\{1\} .
$$

Thus the isomorphism (4-9) holds for $q=1$. This finishes the proof.

Example 4.9 In this example, we discuss the exceptional case by determining the center of the group

$$
G=\left(P_{3} *_{F\left[S^{1}\right]_{2}} P_{3}\right) /\left[R_{i, j} \mid 1 \leq i<j \leq 3\right]_{S},
$$

where $\alpha=A_{1,2}^{m}$ with some $m \neq 0$. By definition, the subgroup $F\left[S^{1}\right]_{2} \leq P_{3}$ is generated by $x_{1}=s_{1} \alpha_{3}=\left(A_{1,3} A_{2,3}\right)^{m}$ and $x_{2}=s_{0} \alpha_{3}=\left(A_{1,2} A_{1,3}\right)^{m}$. Thus the free product with amalgamation $P_{3} *{ }_{F}\left[S^{1}\right]_{2} P_{3}$ is given as the quotient group of $P_{3} * P_{3}$ by the new relations:

$$
\left(A_{1,3} A_{2,3}\right)^{m}=\left(A_{1,3}^{\prime} A_{2,3}^{\prime}\right)^{m} \quad \text { and } \quad\left(A_{1,2} A_{1,3}\right)^{m}=\left(A_{1,2}^{\prime} A_{1,3}^{\prime}\right)^{m} .
$$

Consider the subgroup $\left[R_{i, j} \mid 1 \leq i<j \leq 3\right]_{S}$ of $P_{3} * F\left[S^{1}\right]_{2} P_{3}$. Observe that

$$
\left[A_{1,2}, A_{1,3}\right],\left[A_{1,2}, A_{2,3}\right],\left[A_{1,3}, A_{2,3}\right] \in\left[R_{i, j} \mid 1 \leq i<j \leq 3\right]_{S},
$$

and the subgroup $\left\langle A_{1,2}, A_{1,3}, A_{2,3}\right\rangle$ is Abelian in $G$. Similarly the subgroup $\left\langle A_{1,2}^{\prime}, A_{1,3}^{\prime}, A_{2,3}^{\prime}\right\rangle$ is Abelian in $G$. Thus $\left(A_{1,2}^{\prime} A_{1,3}^{\prime}\right)^{m}=\left(A_{1,2}^{\prime}\right)^{m}\left(A_{1,3}^{\prime}\right)^{m}$ in $G$ and from equation (4-10)

$$
\left(A_{1,2}^{\prime}\right)^{m}=\left(A_{1,2}\right)^{m}\left(A_{1,3}\right)^{m}\left(A_{1,3}^{\prime}\right)^{-m} .
$$


It follows that $A_{1,2}^{\prime}$ commutes with $A_{1,2}$ since $A_{1,2}$ commutes with $\left(A_{1,2}\right)^{m},\left(A_{1,3}\right)^{m}$ and $\left(A_{1,3}^{\prime}\right)^{m}$. From this, we conclude that $\left(A_{1,2}^{\prime}\right)^{m} \in Z(G)$ because $A_{1,2}^{\prime}$ commutes with all of the generators for $G$. Similarly

$$
\left(A_{1,2}\right)^{m},\left(A_{1,3}\right)^{m},\left(A_{2,3}\right)^{m},\left(A_{1,3}^{\prime}\right)^{m},\left(A_{2,3}^{\prime}\right)^{m} \in Z(G) .
$$

Thus the subgroup

$$
H=\left\langle\left(\left(A_{1,2}\right)^{m},\left(A_{1,3}\right)^{m},\left(A_{2,3}\right)^{m},\left(A_{1,2}^{\prime}\right)^{m},\left(A_{1,3}^{\prime}\right)^{m},\left(A_{2,3}^{\prime}\right)^{m}\right\rangle \leq Z(G) .\right.
$$

Let

$$
\begin{aligned}
G^{\prime}=\left(\mathbb{Z}\left(A_{1,2}\right) / m * \mathbb{Z}\left(A_{1,2}^{\prime}\right) / m\right) \times\left(\mathbb{Z}\left(A_{1,3}\right) / m * \mathbb{Z}\left(A_{1,3}^{\prime}\right) / m\right) \\
\times\left(\mathbb{Z}\left(A_{2,3}\right) / m * \mathbb{Z}\left(A_{2,3}^{\prime}\right) / m\right)
\end{aligned}
$$

and let $\phi: P_{3} * P_{3} \rightarrow G^{\prime}$ be the canonical quotient homomorphism defined by sending generators to generators. Then

$$
\phi\left(x_{1}\right)=\phi\left(x_{2}\right)=1 .
$$

Moreover $\phi\left(\left[R_{i, j} \mid 1 \leq i<j \leq 3\right]_{S}\right)=1$ with $\phi(H)=1$ and so $\phi$ induces an epimorphism $\bar{\phi}$ in the following diagram:

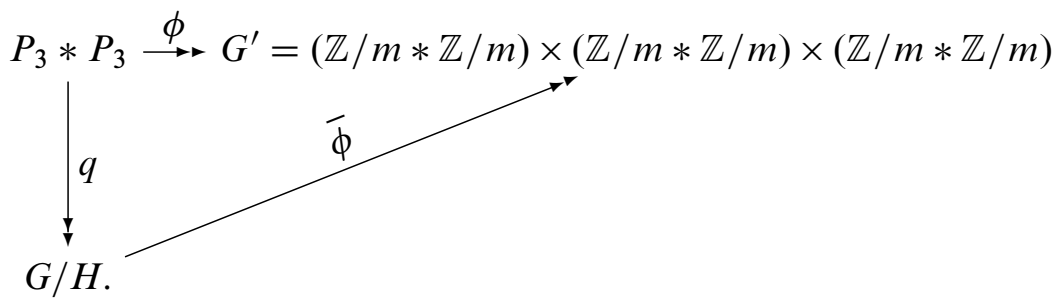

On the other hand, the group homomorphism

$$
\mathbb{Z}\left(A_{1,2}\right) * \mathbb{Z}\left(A_{1,2}^{\prime}\right) \longrightarrow G / H
$$

factors through the quotient $\mathbb{Z}\left(A_{1,2}\right) / m * \mathbb{Z}\left(A_{1,2}^{\prime}\right) / m$. Similarly there are canonical group homomorphisms from $\mathbb{Z}\left(A_{1,3}\right) / m * \mathbb{Z}\left(A_{1,3}^{\prime}\right) / m$ and $\mathbb{Z}\left(A_{1,2}\right) / m * \mathbb{Z}\left(A_{1,2}^{\prime}\right) / m$ to $G / H$. Since the subgroups $\left\langle A_{1,2}, A_{1,2}^{\prime}\right\rangle,\left\langle A_{1,3}, A_{1,3}^{\prime}\right\rangle$ and $\left\langle A_{2,3}, A_{2,3}^{\prime}\right\rangle$ commute with each other in the group $G$, there is a group epimorphism

$$
\psi: G^{\prime} \rightarrow G / H
$$

such that $\bar{\phi} \circ \psi=\mathrm{id}_{G^{\prime}}$ since all of generators of $G / H$ lie in the image of $\psi$. It follows that

$$
G / H \cong G^{\prime}=(\mathbb{Z} / m * \mathbb{Z} / m) \times(\mathbb{Z} / m * \mathbb{Z} / m) \times(\mathbb{Z} / m * \mathbb{Z} / m)
$$


Since $Z\left(G^{\prime}\right)=\{1\}, Z(G / H)=\{1\}$ and so

$$
Z(G) \leq H .
$$

Together with equation (4-11), we have $Z(G)=H \cong \mathbb{Z}^{\oplus 4}$.

\section{Description of homotopy groups of the Moore spaces $M(\mathbb{Z} / q, k)$ with $k \geq 3$}

In this section, we give an explicit combinatorial description of the homotopy groups of the Moore spaces $M(\mathbb{Z} / q, k)$ with $k \geq 3$. This description highlights our methodology for giving combinatorial descriptions of homotopy groups using free products of braid groups.

\subsection{An embedding of $F\left[S^{k-1}\right]$ into $\mathcal{T}\left(S^{k} ; \alpha\right)$ for Moore boundaries $\alpha$}

Let $\tilde{\alpha} \in N_{k-1} F\left[S^{1}\right]$ with $d_{0} \tilde{\alpha} \neq 1$. We are going to construct a simplicial monomorphism $F\left[S^{k-1}\right] \rightarrow \mathcal{T}\left(S^{k} ; d_{0} \tilde{\alpha}\right)$, which is also a homotopy equivalence.

Let

$$
f_{\widetilde{\alpha}}: \Delta[k-1] \longrightarrow F\left[S^{1}\right]
$$

be the representing map of the element $\tilde{\alpha}$ with $f_{\widetilde{\alpha}}\left(\sigma_{k}\right)=\tilde{\alpha}$, where $\sigma_{k}=(0,1, \ldots$, $k-1) \in \Delta[k-1]$. Let $\Lambda^{0}[k-1]$ be the simplicial subset of $\Delta[k-1]$ generated by $d_{j} \sigma_{k-1}$ for $j>0$ and let

$$
\bar{\Delta}[k-1]=\Delta[k-1] / \Lambda^{0}[k-1] .
$$

Since $d_{j} \widetilde{\alpha}=1$ for $j>0$, the simplicial map $f_{\widetilde{\alpha}}$ factors through the simplicial quotient $\bar{\Delta}[k-1]$. Let

$$
\bar{f}_{\widetilde{\alpha}}: \bar{\Delta}[k-1] \longrightarrow F\left[S^{1}\right]
$$

be the resulting simplicial map with $\bar{f}_{\widetilde{\alpha}}\left(\sigma_{k-1}\right)=\widetilde{\alpha}$. By the universal property of Milnor's construction, there exists a unique simplicial homomorphism

$$
\theta_{\tilde{\alpha}}: F[\bar{\Delta}[k-1]] \longrightarrow F\left[S^{1}\right]
$$

such that $\left.\theta_{\tilde{\alpha}}\right|_{\bar{\Delta}[k-1]}=\bar{f}_{\widetilde{\alpha}}$.

Lemma 5.1 The simplicial group $F[\bar{\Delta}[k-1]]$ is contractible and the map

$$
\theta_{\tilde{\alpha}}: F[\bar{\Delta}[k-1]] \longrightarrow F\left[S^{1}\right]
$$

is a simplicial monomorphism. 
Proof Recall [10] that the geometric realization $|\Delta[k-1]|$ is the standard $(k-1)-$ simplex $\Delta^{k-1}$ and $\left|\Lambda^{0}[k]\right|$ is the union of all faces of $\Delta^{k-1}$ except the first face. Thus both $|\Delta[k-1]|$ and $\left|\Lambda^{0}[k]\right|$ are contractible and so is $|\bar{\Delta}[k-1]|=\left|\Delta[k-1] / \Lambda^{0}[k-1]\right|$. It follows that

$$
|F[\bar{\Delta}[k-1]]| \simeq \Omega \Sigma|\bar{\Delta}[k-1]|
$$

is contractible.

The proof of the statement regarding $\theta_{\tilde{\alpha}}$ is similar to that of Lemma 4.2. The image $\theta_{\tilde{\alpha}}(F[\bar{\Delta}[k-1]])$ is a simplicial free group because it is a simplicial subgroup of the simplicial free group $F\left[S^{1}\right]$. Following the lines in the proof of Lemma 4.2, for checking that $\theta_{\tilde{\alpha}}: F[\bar{\Delta}[k-1]] \rightarrow \theta_{\tilde{\alpha}}(F[\bar{\Delta}[k-1]])$ is a simplicial monomorphism, it suffices to show that

$$
N \theta_{\widetilde{\alpha}}^{\mathrm{ab}}: N_{*} F[\bar{\Delta}[k-1]]^{\mathrm{ab}} \longrightarrow N_{*} \theta_{\widetilde{\alpha}}(F[\bar{\Delta}[k-1]])
$$

is an isomorphism. This follows directly from the computations that

$$
\begin{array}{r}
N_{q} F[\bar{\Delta}[k-1]]^{\mathrm{ab}}= \begin{cases}\mathbb{Z}\left(\sigma_{k-1}\right) & \text { if } q=k-1, \\
\mathbb{Z}\left(d_{0} \sigma_{k-1}\right) & \text { if } q=k-2, \\
0 & \text { otherwise, }\end{cases} \\
N_{q} \theta_{\tilde{\alpha}}\left(F[\bar{\Delta}[k-1])^{\mathrm{ab}}= \begin{cases}\mathbb{Z}(\widetilde{\alpha}) & \text { if } q=k-1, \\
\mathbb{Z}\left(d_{0} \tilde{\alpha}=\alpha\right) & \text { if } q=k-2, \\
0 & \text { otherwise, }\end{cases} \right.
\end{array}
$$

and $\theta_{\tilde{\alpha}}\left(\sigma_{k-1}\right)=\tilde{\alpha}$.

Now from the above lemma, the simplicial monomorphism

$$
\phi_{\alpha}: F\left[S^{k-2}\right] \longrightarrow \mathrm{AP}_{*}
$$

is given by the composite:

$$
F\left[S^{k-2}\right] \stackrel{\iota}{\hookrightarrow} F[\bar{\Delta}[k-1]] \stackrel{\theta_{\widetilde{\alpha}}}{\longrightarrow} F\left[S^{1}\right] \stackrel{\Theta}{\hookrightarrow} \mathrm{AP}_{*}
$$

It follows that $\Theta \circ \theta_{\tilde{\alpha}}: F[\bar{\Delta}[k-1]] \rightarrow \mathrm{AP}_{*}$ induces a simplicial monomorphism

$$
F[\bar{\Delta}[k-1]] *_{F\left[S^{k-2}\right]} F[\bar{\Delta}[k-1]] \longleftrightarrow \mathrm{AP}_{*} *_{F\left[S^{k-2}\right]} \mathrm{AP}_{*}
$$

which is a homotopy equivalence by Theorem 3.2. Let $\sigma_{k-1}^{\prime}$ denote the element $\sigma_{k-1}$ in second copy of $F[\bar{\Delta}[k-1]]$ in the free product with amalgamation

$$
F[\bar{\Delta}[k-1]] *_{F\left[S^{k-2}\right]} F[\bar{\Delta}[k-1]] .
$$

Let

$$
z_{k-1}=\sigma_{k-1}\left(\sigma_{k-1}^{\prime}\right)^{-1} \in\left(F[\bar{\Delta}[k-1]] *_{F\left[S^{k-2}\right]} F[\bar{\Delta}[k-1]]\right)_{k-1} .
$$


Then $z_{k-1}$ is a Moore cycle because

$$
d_{j} z_{k-1}=d_{j} \sigma_{k-1}\left(d_{j} \sigma_{k-1}^{\prime}\right)^{-1}=1
$$

for $j>0$ in $F[\bar{\Delta}[k-1]] * F\left[S^{k-2}\right] F[\bar{\Delta}[k-1]]$ and

$$
d_{0} z_{k-1}=d_{0} \sigma_{k-1}\left(d_{0} \sigma_{k-1}^{\prime}\right)^{-1}=1
$$

since $d_{0} \sigma_{k-1}=d_{0} \sigma_{k-1}^{\prime}$ lies in the amalgamated subgroup $F\left[S^{k-2}\right]$. Let

$$
f_{z_{k-1}}: S^{k-1} \rightarrow F[\bar{\Delta}[k-1]] *_{F\left[S^{k-2}\right]} F[\bar{\Delta}[k-1]]
$$

be the representing map of $z_{k-1}$ and let

$$
\tilde{f}_{z_{k-1}}: F\left[S^{k-1}\right] \longrightarrow F[\bar{\Delta}[k-1]] *_{F\left[S^{k-2}\right]} F[\bar{\Delta}[k-1]]
$$

be the simplicial homomorphism induced by $f_{z_{k-1}}$.

Lemma 5.2 Let $\tilde{f}_{z_{k-1}}$ be defined as above. Then:

(1) $\tilde{f}_{z_{k-1}}$ is a simplicial monomorphism.

(2) $\tilde{f}_{z_{k-1}}$ is a homotopy equivalence.

Proof (1) Observe that

$$
F[\bar{\Delta}[k-1]] *{ }_{F\left[S^{k-2}\right]} F[\bar{\Delta}[k-1]]=F[\bar{\Delta}[k-1] \cup \bar{\Delta}[k-1]]
$$

is a simplicial free group, where $\bar{\Delta}[k-1] \cup \bar{\Delta}[k-1]$ is the simplicial union by identification $d_{0} \sigma_{k-1}$ with $d_{0} \sigma_{k-1}^{\prime}$. Assertion (1) follows from the lines of the proof of Lemma 4.2.

(2) Since

$$
\tilde{f}_{z_{k-1}}: F\left[S^{k-1}\right] \simeq \Omega S^{k} \longrightarrow F[\bar{\Delta}[k-1]] * F\left[S^{k-2}\right] F[\bar{\Delta}[k-1]] \simeq \Omega S^{k}
$$

is a simplicial homomorphism, it is a loop map. Thus it suffices to show that $\tilde{f}_{z_{k-1}}$ induces an isomorphism

$$
\tilde{f}_{z_{k-1} *}: \pi_{k-1}\left(F\left[S^{k-1}\right]\right) \cong \mathbb{Z} \longrightarrow \pi_{k-1}\left(F[\bar{\Delta}[k-1]] *{ }_{F\left[S^{k-2}\right]} F[\bar{\Delta}[k-1]]\right) \cong \mathbb{Z} .
$$

Note that:

$$
\pi_{k-1}\left(F[\bar{\Delta}[k-1]] *_{F\left[S^{k-2}\right]} F[\bar{\Delta}[k-1]]\right) \cong \pi_{k-1}\left(F[\bar{\Delta}[k-1] \cup \bar{\Delta}[k-1]]^{\mathrm{ab}}\right)
$$


Now the Moore chain complex of $F[\bar{\Delta}[k-1] \cup \bar{\Delta}[k-1]]^{\text {ab }}$ is given by

$$
N_{q} F[\bar{\Delta}[k-1] \cup \bar{\Delta}[k-1]]^{\mathrm{ab}}= \begin{cases}\mathbb{Z}\left(\sigma_{k-1}\right) \oplus \mathbb{Z}\left(\sigma_{k-1}^{\prime}\right) & \text { if } q=k-1, \\ \mathbb{Z}\left(d_{0} \sigma_{k-1}=d_{0} \sigma_{k-1}^{\prime}\right) & \text { if } q=k-2, \\ 0 & \text { otherwise. }\end{cases}
$$

Thus $\pi_{k-1}\left(F[\bar{\Delta}[k-1] \cup \bar{\Delta}[k-1]]^{\mathrm{ab}}\right)$ is generated by $\sigma_{k-1}-\sigma_{k-1}^{\prime}$, which is the image of $z_{k-1}$ in the Abelianization $F[\bar{\Delta}[k-1] \cup \bar{\Delta}[k-1]]^{\mathrm{ab}}$. It follows that

$$
\tilde{f}_{z_{k-1} *}: \pi_{k-1}\left(F\left[S^{k-1}\right]\right) \longrightarrow \pi_{k-1}\left(F[\bar{\Delta}[k-1]] *_{F\left[S^{k-2}\right]} F[\bar{\Delta}[k-1]]\right)
$$

is an isomorphism and hence the result.

\subsection{Description for $\pi_{*}(M(\mathbb{Z} / q, k))$ with $k \geq 3$}

With the preparation in the previous subsection, we can now construct a simplicial group model for $\Omega M(\mathbb{Z} / q, k)$ with $k \geq 3$. Let $\alpha \in \mathcal{Z}_{k-1} F\left[S^{1}\right]$ be a Moore cycle with $\alpha \neq 1$ and let $\tilde{\alpha} \in N_{k-1} F\left[S^{1}\right]$ be a Moore chain such that $d_{0} \tilde{\alpha} \neq 1$. From Lemma 5.2 together with isomorphism (5-3), there is a simplicial monomorphism

$$
\delta_{\tilde{\alpha}}: F\left[S^{k-1}\right] \longrightarrow \mathcal{T}\left(S^{k} ; d_{0} \tilde{\alpha}\right),
$$

which is a homotopy equivalence. Let

$$
F[q]: F\left[S^{k-1}\right] \longrightarrow F\left[S^{k-1}\right]
$$

be the simplicial homomorphism such that

$$
F[q](x)=x^{q}
$$

for $x \in S^{k-1} \subseteq F\left[S^{k-1}\right]$. Clearly $F[q]$ is a simplicial monomorphism. Now define the simplicial group $\mathcal{T}(M(\mathbb{Z} / q, k) ; \alpha)$ to be the free product with amalgamation:

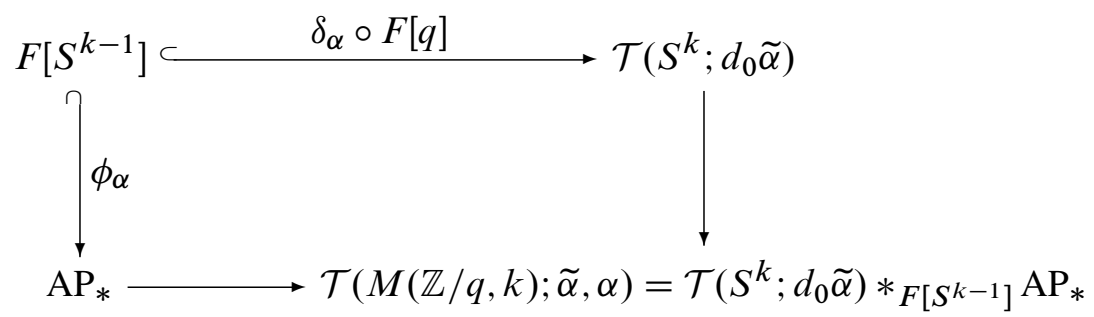

The construction of $\delta_{\widetilde{\alpha}} \circ F[q]$ is explicitly given as follows:

Regard $\widetilde{\alpha}$ as in $k$-strand braid through the embedding $\Theta: F\left[S^{1}\right] \rightarrow \mathrm{AP}_{*}$. Let $\widetilde{\alpha}^{\prime}$ be a copy of $\widetilde{\alpha}$ for the second copy of $\mathrm{AP}_{*}$ in the free product with amalgamation:

$$
\mathcal{T}\left(S^{k} ; d_{0} \tilde{\alpha}\right)=\mathrm{AP}_{*} * F\left[S^{k-2}\right] \mathrm{AP}_{*}
$$


Let $\sigma_{k-1}$ be the non-degenerate element in $S_{k-1}^{k-1}$. Then

$$
\delta_{\widetilde{\alpha}} \circ F[q]: F\left[S^{k-1}\right] \rightarrow \mathcal{T}\left(S^{k} ; d_{0} \tilde{\alpha}\right)
$$

is the unique simplicial homomorphism such that $\delta\left(\sigma_{k-1}\right)=\left(\widetilde{\alpha}\left(\widetilde{\alpha}^{\prime}\right)^{-1}\right)^{q}$. In the language of braids, $\delta_{\tilde{\alpha}} \circ F[q]\left(F\left[S^{k-1}\right]\right)$ is the subgroup of $\mathcal{T}\left(S^{k} ; d_{0} \tilde{\alpha}\right)=$ $\mathrm{AP}_{*} * F\left[S^{k-2}\right] \mathrm{AP}_{*}$ generated by the cablings of $\left(\widetilde{\alpha}\left(\widetilde{\alpha}^{\prime}\right)^{-1}\right)^{q}$ in the self free product with amalgamation of braid groups.

One interesting point in the simplicial group

$$
\mathcal{T}(M(\mathbb{Z} / q, k) ; \widetilde{\alpha}, \alpha)=\left(\mathrm{AP}_{*} *_{F\left[S^{k-2}\right]} \mathrm{AP}_{*}\right) *_{F\left[S^{k-1}\right]} \mathrm{AP}_{*}
$$

is that we identify the $q^{\text {th }}$ power $\left(\widetilde{\alpha}\left(\widetilde{\alpha}^{\prime}\right)^{-1}\right)^{q} \in \mathrm{AP}_{*} * F\left[S^{k-2}\right] \mathrm{AP}_{*}$ with $\alpha \in \mathrm{AP}_{*}$. So the cablings of $\alpha$ have $q^{\text {th }}$ roots in $\mathcal{T}(M(\mathbb{Z} / q, k) ; \tilde{\alpha}, \alpha)$.

Theorem 5.3 Let $\alpha \in \mathcal{Z}_{k-1} F\left[S^{1}\right]$ be a Moore cycle with $\alpha \neq 1$ and let $\tilde{\alpha} \in$ $N_{k-1} F\left[S^{1}\right]$ be a Moore chain such that $d_{0} \tilde{\alpha} \neq 1$. Then the simplicial group

$$
\mathcal{T}(M(\mathbb{Z} / q, k) ; \tilde{\alpha}, \alpha)
$$

is homotopy equivalent to the loop space $\Omega M(\mathbb{Z} / q, k)$ of the Moore space. Moreover the canonical inclusion

$$
\mathcal{T}\left(S^{k} ; d_{0} \tilde{\alpha}\right) \hookrightarrow \mathcal{T}(M(\mathbb{Z} / q, k) ; \tilde{\alpha}, \alpha)
$$

is homotopic to the looping of the inclusion $S^{k} \hookrightarrow M(\mathbb{Z} / q, k)$.

Proof By Theorem 3.2, the classifying space $\bar{W}(\mathcal{T}(M(\mathbb{Z} / q, k) ; \widetilde{\alpha}, \alpha))$ is given by the homotopy push-out

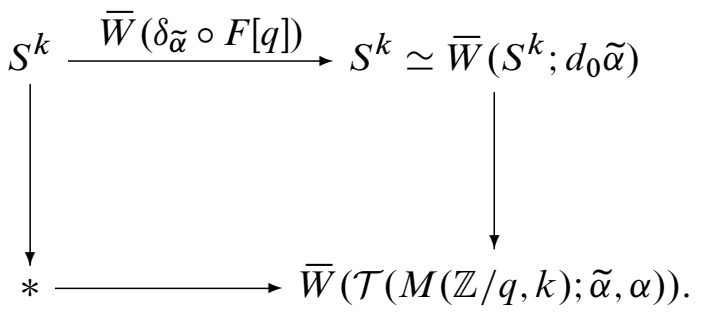

Since

$$
\bar{W}\left(\delta_{\widetilde{\alpha}} \circ F[q]_{*}\right): \pi_{k}\left(S^{k}\right) \cong \pi_{k-1}\left(F\left[S^{k-1}\right]\right) \longrightarrow \pi_{k}\left(S^{k}\right) \cong \pi_{k-1}\left(\mathcal{T}\left(S^{k} ; d_{0} \widetilde{\alpha}\right)\right)
$$

is of degree $q, \bar{W}(\mathcal{T}(M(\mathbb{Z} / q, k) ; \tilde{\alpha}, \alpha)) \simeq M(\mathbb{Z} / q, k)$. Observe that the right column of the above diagram is homotopic to the inclusion of the bottom cell $S^{k} \hookrightarrow M(\mathbb{Z} / q, k)$. The assertions follow. 
Let $A_{i, j}, A_{i, j}^{\prime}$ and $A_{i, j}^{\prime \prime}$ be copies of $A_{i, j}$ for generators for $P_{n}$ in the free product with amalgamation

$$
\mathcal{T}(M(\mathbb{Z} / q, k) ; \tilde{\alpha}, \alpha)_{n-1}=\left(P_{n} *_{F\left[S^{k-2}\right]_{n-1}} P_{n}\right) *_{F\left[S^{k-1}\right]_{n-1}} P_{n}
$$

and let $R_{i, j}$ be the normal closure of $A_{i, j}, A_{i, j}^{\prime}$ and $A_{i, j}^{\prime \prime}$ in $\mathcal{T}(M(\mathbb{Z} / q, k) ; \tilde{\alpha}, \alpha)_{n-1}$.

Theorem 5.4 Let $k \geq 3$. Let $\alpha \in \mathcal{Z}_{k-1} F\left[S^{1}\right]$ be a Moore cycle with $\alpha \neq 1$ and let $\tilde{\alpha} \in N_{k-1} F\left[S^{1}\right]$ be a Moore chain such that $d_{0} \tilde{\alpha} \neq 1$. Then $\pi_{n}(M(\mathbb{Z} / q, k))$ is isomorphic to the center of the group

$$
\left(\left(P_{n} *_{F\left[S^{k-2}\right]_{n-1}} P_{n}\right) *_{F\left[S^{k-1}\right]_{n-1}} P_{n}\right) /\left[R_{i, j} \mid 1 \leq i<j \leq n\right]_{S}
$$

for any $n$.

Proof Since $\mathcal{T}(M(\mathbb{Z} / q, k) ; \tilde{\alpha}, \alpha)$ is a simplicial quotient group of $\mathcal{G} * \mathcal{G} * \mathcal{G}$, the Moore boundaries

$$
\mathcal{B}_{n-1} \mathcal{T}(M(\mathbb{Z} / q, k) ; \tilde{\alpha}, \alpha)=\left[R_{i, j} \mid 1 \leq i<j \leq n\right]_{S}
$$

by Lemma 4.5. Observe that the group $\left(P_{m} *_{F\left[S^{k-2}\right]_{m-1}} P_{n}\right) *_{F\left[S^{k-1}\right]_{m-1}} P_{m}$ has trivial center by Lemma 4.7. The assertion follows from [24, Proposition 2.14].

Remark 5.5 An explicit choice of $\alpha$ and $\tilde{\alpha}$ can be given. For instance, we can choose

$$
\alpha_{k+1}=\left[\ldots\left[\left[\xi_{1}^{-1}, \xi_{1} \xi_{2}^{-1}\right], \xi_{2} \xi_{3}^{-1}\right], \ldots, \xi_{k-2} \xi_{k-1}^{-1}, \xi_{k-1}\right]
$$

in Theorem 2.2 as a $k$-strand Brunnian braid and choose

$$
\tilde{\alpha}_{k}=\left[\ldots\left[\xi_{1} \xi_{2}^{-1}, \xi_{2} \xi_{3}^{-1}\right], \ldots, \xi_{k-2} \xi_{k-1}^{-1}, \xi_{k-1}\right]
$$

as a $k$-strand quasi-Brunnian braid in the sense of [7], ie, a braid which becomes trivial after deleting any strand except possibly for the first strand. Here the $\xi_{i}$ are elements of $P_{k}$ described in the introduction (see Step 1, page 2). Then we obtain an explicit simplicial group model $\mathcal{T}\left(M(\mathbb{Z} / q, k) ; \widetilde{\alpha}_{k}, \alpha_{k+1}\right)$ for $\Omega M(\mathbb{Z} / q, k)$. 


\section{Description of the homotopy groups of Moore spaces $M(\mathbb{Z} / q, 2)$ and proof of Theorem 2.3}

Let $\mathcal{T}(M(\mathbb{Z} / q, 2))$ be the free product with amalgamation by the following diagram:

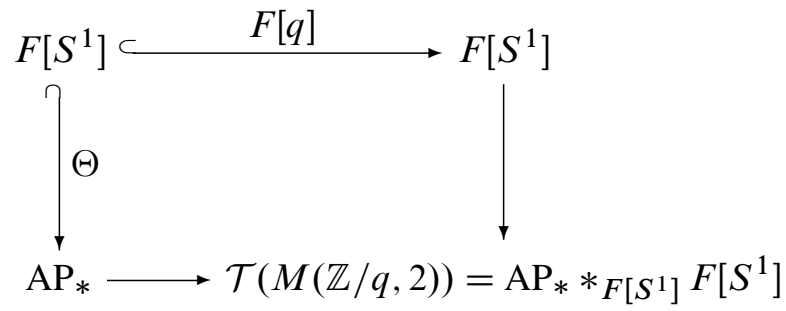

By Theorem 3.2, there is a homotopy push-out

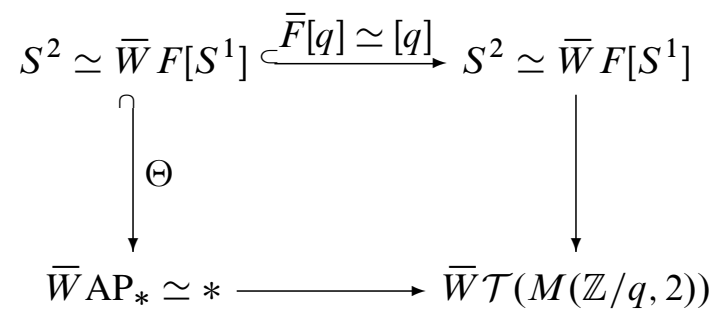

and so $\bar{W} \mathcal{T}(M(\mathbb{Z} / q, 2) \simeq M(\mathbb{Z} / q, 2)$. Namely $\mathcal{T}(M(\mathbb{Z} / q, 2))$ is a simplicial group model for $\Omega M(\mathbb{Z} / q, 2)$.

For each $n$, the homomorphism

$$
F[q]: F\left[S^{1}\right]_{n-1}=F_{n-1} \longrightarrow F\left[S^{1}\right]_{n-1}=F_{n-1}
$$

is the homomorphism $\phi_{q}$ described in Theorem 2.3. Thus as a group

$$
\mathcal{T}(M(\mathbb{Z} / q, 2))_{n-1}=P_{n} *_{\phi_{q}} F_{n-1} .
$$

We give an more explicit description of the group $\mathcal{T}(M(\mathbb{Z} / q, 2))_{n-1}$ using degeneracy operations. Let $\left\{x_{j}\right\}_{j=1, \ldots, n-1}$ be the set of generators for $F_{n-1}=F\left[S^{1}\right]_{n-1}$ as the second factor in the free product $P_{n} *_{\phi_{q}} F_{n-1}$ for $1 \leq j \leq n-1$. (Note: In the introduction to Theorem 2.3, we write $y_{j}$ for $x_{j}$.) As an element in $F\left[S^{1}\right]_{n-1}$,

$$
x_{j}=s_{n-2} \cdots s_{j+1} s_{j} s_{j-2} s_{j-3} \cdots s_{1} s_{0} \sigma_{1}
$$

for $1 \leq j \leq n-1$. The group $\mathcal{T}(M(\mathbb{Z} / q, 2))_{n-1}$ is the quotient group of $P_{n} * F_{n-1}$ by the relation

$$
s_{j+1} s_{j} s_{j-2} s_{j-3} \cdots s_{1} s_{0} A_{1,2}=x_{j}^{q}
$$


for $1 \leq j \leq n-1$, where $s_{j+1} s_{j} s_{j-2} s_{j-3} \cdots s_{1} s_{0} A_{1,2}$ is the cabling of $A_{1,2}$ as in the picture in the introduction.

Let $z_{1}=x_{1}, z_{n}=x_{n-1}$ and $z_{i}=x_{i} x_{i-1}^{-1}$, for $i=2, \ldots, n-1$. Now let $R_{i}=$ $\left\langle z_{i}\right\rangle^{P_{n} *_{\phi_{q}} F_{n-1}}$ be the normal closure of $z_{i}$ in $P_{n} *_{\phi_{q}} F_{n-1}$ for $1 \leq i \leq n$ and let $R_{s, t}=\left\langle A_{s, t}\right\rangle P_{n} *_{\phi_{q}} F_{n-1}$ be the normal closure of $A_{s, t}$ in $P_{n} *_{\phi_{q}} F_{n-1}$ for $1 \leq s<t \leq n$. Define the index set $\operatorname{Index}\left(R_{j}\right)=\{j\}$ for $1 \leq j \leq n$ and $\operatorname{Index}\left(R_{s, t}\right)=\{s, t\}$ for $1 \leq s<t \leq n$. Now define the symmetric commutator subgroup

$$
\left[R_{i}, R_{s, t} \mid 1 \leq i \leq n, 1 \leq s<t \leq n\right]_{S}=\underset{\{1,2, \ldots, n\}=\bigcup_{j=1}^{t} \operatorname{Index}\left(C_{j}\right)}{\left[\ldots\left[C_{1}, C_{2}\right], \ldots, C_{t}\right]}
$$

where each $C_{j}=R_{i}$ or $R_{s, t}$ for some $i$ or $(s, t)$.

Theorem 6.1 (Theorem 2.3) The homotopy group $\pi_{n}(M(\mathbb{Z} / q, 2)$ ) is isomorphic to the center of the group

$$
\left(P_{n} * \phi_{q} F_{n-1}\right) /\left[R_{i}, R_{s, t} \mid 1 \leq i \leq n, 1 \leq s<t \leq n\right]_{S}
$$

for any $n>3$.

Proof The proof is similar to that of Theorem 2.2. It is easy to see that the group $\mathcal{T}(M(\mathbb{Z} / q, 2))_{m}=P_{m+1} *_{\phi_{q}} F_{m}$ has the trivial center for $m \geq 2$. From [24, Proposition 2.14], $\pi_{m}(\mathcal{T}(M(\mathbb{Z} / q, 2))) \cong \pi_{m+1}(M(\mathbb{Z} / q, 2))$ is isomorphic to the center of $\mathcal{T}(M(\mathbb{Z} / 2))_{m} / \mathcal{B}_{m} \mathcal{T}(M(\mathbb{Z} / q, 2))$ for $m \geq 3$. Thus the key point is to show the Moore boundaries

$$
\mathcal{B}_{n-1} \mathcal{T}(M(\mathbb{Z} / q, 2))=\left[R_{i}, R_{s, t} \mid 1 \leq i \leq n, 1 \leq s<t \leq n\right]_{S} .
$$

We construct a simplicial group $\tilde{F}$ by $\widetilde{F}_{n-1}$ generated by the letters $z_{1}, \ldots, z_{n}$ with face operation

$$
d_{j} z_{k}= \begin{cases}z_{k} & \text { if } k<j+1, \\ 1 & \text { if } k=j+1, \\ z_{k-1} & \text { if } k>j+1,\end{cases}
$$

and degeneracy operations

$$
s_{j} z_{k}= \begin{cases}z_{k} & \text { if } k<j+1, \\ z_{j+1} z_{j+2} & \text { if } k=j+1 \\ z_{k+1} & \text { if } k>j+1\end{cases}
$$

for $0 \leq j \leq n-1$. Then $\widetilde{F}$ is a simplicial group with a simplicial epimorphism $f: \widetilde{F} \rightarrow F\left[S^{1}\right]$ by sending the letter $z_{j}$ of $\widetilde{F}_{n-1}$ to the element $z_{j} \in F\left[S^{1}\right]_{n-1}$. Let 
$g: \mathcal{G} \rightarrow \mathrm{AP}_{*}$ be the canonical simplicial epimorphism. Then we have the simplicial epimorphism:

$$
\mathcal{G} * \widetilde{F} \longrightarrow \mathrm{AP}_{*} * F\left[S^{1}\right] \longrightarrow \mathcal{T}(M(\mathbb{Z} / q, 2))
$$

Observe that $\operatorname{Ker}\left(d_{n}:(\mathcal{G} * \widetilde{F})_{n} \rightarrow(\mathcal{G} * \widetilde{F})_{n-1}\right)$ is the normal closure of the elements $x_{i, n+1}, z_{n+1}$. By repeating the arguments in the proof of Lemma 4.5, we have

$$
\mathcal{B}_{n-1}(\mathcal{G} * \tilde{F})=\left[R_{i}, R_{S, t} \mid 1 \leq i \leq n, 1 \leq s<t \leq n\right]_{S}
$$

and hence the result.

Example Consider the case $n=3$. The group

$$
G=\left(P_{3} *_{\phi_{q}} F_{2}\right) /\left[R_{i}, R_{s, t} \mid 1 \leq i \leq 3,1 \leq s<t \leq 3\right]_{S}
$$

is given by generators $x_{1}, x_{2}, a_{12}, a_{13}, a_{23}$ and the following relations:

$$
\begin{gathered}
x_{1}^{q}=a_{12} a_{13}, \quad x_{2}^{q}=a_{13} a_{23}, \\
{\left[\left[x_{1}^{g_{1}}, x_{2}^{g_{2}}\right], x_{1}\right]=\left[\left[x_{1}^{g_{1}}, x_{2}^{g_{2}}\right], x_{2}\right]=1, g_{1}, g_{2} \in G} \\
{\left[a_{12}^{g}, a_{13}\right]=\left[a_{12}^{g}, a_{23}\right]=\left[a_{13}^{g}, a_{23}\right]=1, g \in G} \\
{\left[x_{1}^{g}, a_{23}\right]=\left[\left(x_{1} x_{2}^{-1}\right)^{g}, a_{13}\right]=\left[x_{2}^{g}, a_{12}\right]=1, g \in G}
\end{gathered}
$$

Presenting $a_{13}, a_{23}$ via generators $x_{1}, x_{2}, a_{12}$, we get the following 3-generator presentation of $G$ :

$$
\begin{gathered}
{\left[\left[x_{1}^{g_{1}}, x_{2}^{g_{2}}\right], x_{1}\right]=\left[\left[x_{1}^{g_{1}}, x_{2}^{g_{2}}\right], x_{2}\right]=1, g_{1}, g_{2} \in G} \\
{\left[a_{12}^{g}, a_{12}^{-1} x_{1}^{q}\right]=\left[a_{12}^{g}, x_{1}^{-q} a_{12} x_{2}^{q}\right]=\left[\left(a_{12}^{-1} x_{1}^{q}\right)^{g}, x_{1}^{-q} a_{12} x_{2}^{q}\right]=1, g \in G} \\
{\left[x_{1}^{g}, x_{1}^{-q} a_{12} x_{2}^{q}\right]=\left[\left(x_{1} x_{2}^{-1}\right)^{g}, a_{12}^{-1} x_{1}^{q}\right]=\left[x_{2}^{g}, a_{12}\right]=1, g \in G}
\end{gathered}
$$

Straightforward computations show that $G$ is a 3-generator nilpotent group of class 2, given by generators $x_{1}, x_{2}, a_{12}$ and relations:

$\left[a_{12}, x_{2}\right]=\left[a_{12}, x_{1}^{q}\right]=\left[x_{1}^{q}, x_{2}^{q}\right]=\left[x_{1}, a_{12} x_{2}^{q}\right]=\left[x_{1} x_{2}^{-1}, a_{12}^{-1} x_{1}^{q}\right]=1, \quad[[G, G], G]=1$

It follows that the order of the element $\left[x_{1}, x_{2}\right]$ is $\left(2 q, q^{2}\right)$ in $G$. The center of $G$ is bigger than the subgroup generated by $\left[x_{1}, x_{2}\right]$, since $a_{12}^{q}$ lies in the center. Denote $Z_{1}=\left\langle a_{12}, x_{1}\right\rangle^{G}, Z_{2}=\left\langle a_{12}, x_{1} x_{2}^{-1}\right\rangle^{G}, Z_{3}=\left\langle x_{2}\right\rangle^{G}$. The homotopy group $\pi_{3} M(Z / q, 2)$ is given now as the intersection

$$
Z_{1} \cap Z_{2} \cap Z_{3} \cong \mathbb{Z} /\left(2 q, q^{2}\right) .
$$




\section{Acknowledgments}

This article was finished during the visit of both authors to Dalian University of Technology under the support of a grant (No. 11028104) of NSFC of China in July of 2011. The authors would like to thank the hospitality of Dalian University of Technology for supporting our research on this topic. The authors are thankful to L Breen, H Miller, S Theriault and the anonymous referee for their valuable comments and suggestions to improve the manuscript.

The research of the first author is supported by the Chebyshev Laboratory (Department of Mathematics and Mechanics, St. Petersburg State University) under RF Government grant 11.G34.31.0026 and the research of the second author is supported in part by the AcRF Tier 1 (WBS No. R-146-000-137-112) and AcRF Tier 2 (WBS No. R146-000-143-112) of MOE of Singapore and a grant (No. 11028104) of NSFC of China.

\section{References}

[1] V G Bardakov, R Mikhailov, V V Vershinin, J Wu, Brunnian braids on surfaces, Algebr. Geom. Topol. 12 (2012) 1607-1648 MR2966697

[2] G Baumslag, Finitely presented groups, from: "Proc. Internat. Conf. Theory of Groups", (L G Kovacs, B H Neumann, editors), Gordon and Breach, New York (1967) 37-50 MR0215902

[3] A J Berrick, F R Cohen, Y L Wong, J Wu, Configurations, braids, and homotopy groups, J. Amer. Math. Soc. 19 (2006) 265-326 MR2188127

[4] A K Bousfield, E B Curtis, D M Kan, D G Quillen, D L Rector, J W Schlesinger, The mod $-p$ lower central series and the Adams spectral sequence, Topology 5 (1966) 331-342 MR0199862

[5] R Brown, J-L Loday, Van Kampen theorems for diagrams of spaces, Topology 26 (1987) 311-335 MR899052

[6] W-L Chow, On the algebraical braid group, Ann. of Math. (2) 49 (1948) 654-658 MR0026050

[7] F R Cohen, J Wu, On braid groups, free groups, and the loop space of the 2-sphere, from: "Categorical decomposition techniques in algebraic topology", (G Arone, J Hubbuck, R Levi, M Weiss, editors), Progr. Math. 215, Birkhäuser, Basel (2004) 93-105 MR2039761

[8] F R Cohen, J Wu, Artin's braid groups, free groups, and the loop space of the 2-sphere, Q. J. Math. 62 (2011) 891-921 MR2853222 
[9] D Conduché, Modules croisés généralisés de longueur 2, from: "Proceedings of the Luminy conference on algebraic $K$-theory”, (E M Friedlander, M Karoubi, editors), volume 34 (1984) 155-178 MR772056

[10] E B Curtis, Simplicial homotopy theory, Advances in Math. 6 (1971) 107-209 MR0279808

[11] G Ellis, R Mikhailov, A colimit of classifying spaces, Adv. Math. 223 (2010) 20972113 MR2601009

[12] A O Houcine, Embeddings in finitely presented groups which preserve the center, J. Algebra 307 (2007) 1-23 MR2278040

[13] D M Kan, A combinatorial definition of homotopy groups, Ann. of Math. 67 (1958) 282-312 MR0111032

[14] D M Kan, On homotopy theory and c.s.s. groups, Ann. of Math. 68 (1958) 38-53 MR0111033

[15] D M Kan, W P Thurston, Every connected space has the homology of a $K(\pi, 1)$, Topology 15 (1976) 253-258 MR0413089

[16] J Y Li, J Wu, On symmetric commutator subgroups, braids, links and homotopy groups, Trans. Amer. Math. Soc. 363 (2011) 3829-3852 MR2775829

[17] W Magnus, A Karrass, D Solitar, Combinatorial group theory: presentations of groups in terms of generators and relations, Pure and Applied Mathematics 13, Interscience Publishers (1966) MR0207802

[18] R Mikhailov, I B S Passi, J Wu, Symmetric ideals in group rings and simplicial homotopy, J. Pure Appl. Algebra 215 (2011) 1085-1092 MR2747240

[19] J Milnor, On the construction $F[K]$, from: "Algebraic topology—a student guide", (J F Adams, editor), London Mathematical Society Lecture Note Series 4, Cambridge Univ. Press (1972) 119-136

[20] D G Quillen, Homotopical algebra, Lecture Notes in Mathematics 43, Springer, Berlin (1967) MR0223432

[21] J H C Whitehead, On the asphericity of regions in a 3-sphere, Fund. Math. 32 (1939) 149-166

[22] J Wu, On combinatorial descriptions of homotopy groups and the homotopy theory of mod 2 Moore spaces, PhD thesis, University of Rochester (1995) MR2693367

[23] J Wu, On fibrewise simplicial monoids and Milnor-Carlsson's constructions, Topology 37 (1998) 1113-1134 MR1650351

[24] J Wu, Combinatorial descriptions of homotopy groups of certain spaces, Math. Proc. Cambridge Philos. Soc. 130 (2001) 489-513 MR1816806

[25] J Wu, A braided simplicial group, Proc. London Math. Soc. 84 (2002) 645-662 MR1888426 
[26] H Zhao, X Wang, Combinatorial description of the homotopy groups of wedge of spheres, Proc. Amer. Math. Soc. 137 (2009) 371-380 MR2439462

St Petersburg Department of Steklov Mathematical Institute and

Chebyshev Laboratory, St Petersburg State University

14th Line, 29b, Saint Petersburg, 199178 Russia

Department of Mathematics, National University of Singapore

2Block S17-06-02, 10 Lower Kent Ridge Road, Singapore 119076, Singapore

romanvm@mi.ras.ru, matwuj@nus.edu.sg

http://www.mi.ras.ru/ romanvm/, http://www.math.nus.edu.sg/ matwujie

Proposed: Haynes Miller

Received: 23 September 2011

Seconded: David Gabai, Bill Dwyer

Revised: 2 October 2012 